\title{
Sub-millimeter observations of the terrestrial atmosphere during an Earth flyby of the MIRO sounder on the Rosetta spacecraft
}

\author{
C. Jiménez ${ }^{\text {a,* }}$, S. Gulkis ${ }^{b}$, G. Beaudin ${ }^{\text {a }}$, T. Encrenaz ${ }^{\text {a }}$, P. Eriksson ${ }^{\text {d }}$, L. Kamp ${ }^{\text {b }}$, S. Lee ${ }^{b}$, \\ S.A. Buehler ${ }^{\mathrm{c}}$, and the MIRO team \\ a Laboratoire d'Etudes du Rayonnement et de la Matiére en Astrophysique, Observatoire de Paris, 61 Avenue de l'Observatoire, 75014 Paris, France \\ ${ }^{\mathrm{b}}$ Jet Propulsion Laboratory, California Institute of Technology, 4800 Oak Grove Drive, MS 169-506, Pasadena, CA 91109, USA \\ ${ }^{c}$ Department of Computer Science, Electrical and Space Engineering, Lulea University of Technology, PO Box 812, 981 28 Kiruna, Sweden \\ d Department of Earth and Space Science, Chalmers University of Technology, SE-412 96 Gothenburg, Sweden
}

\section{A R T I C L E I N F O}

\section{Article history:}

Received 8 December 2012

Received in revised form

19 February 2013

Accepted 27 March 2013

Available online 18 April 2013

Keywords:

Microwave sounder

Cometary observations

Gravitational flyby

Terrestrial atmospheric temperature

Atmospheric radiative transfer

Inversion theory

\begin{abstract}
A B S T R A C T
Sub-millimeter spectra recorded by the MIRO sounder aboard the Rosetta spacecraft have been used at the time of an Earth flyby (November 2007) to check the consistency and validity of the instrumental data. High-resolution spectroscopic data were recorded in 8 channels in the vicinity of the strong water line at $557 \mathrm{GHz}$, and in a broad band continuum channel at $570 \mathrm{GHz}$. An atmospheric radiative transfer code (ARTS) and standard terrestrial atmospheres have been used to simulate the expected observational results. Differences with the MIRO spectra suggest an anomaly in the behavior of four spectroscopic channels. Further technical investigations have shown that a large part of the anomalies are associated with an instability of one of the amplifiers. The quality of the MIRO data has been further tested by inverting the spectra with an atmospheric inversion tool (Qpack) in order to derive a mesospheric temperature profile. The retrieved profile is in good agreement with the one inferred from the Earth Observing System Microwave Limb Sounder (EOS-MLS). This work illustrates the interest of validating instruments aboard planetary or cometary spacecraft by using data acquired during Earth flybys.
\end{abstract}

(c) 2013 Elsevier Ltd. All rights reserved.

\section{Introduction}

The Rosetta spacecraft was launched by the European Space Agency on 2 March 2004, with the purpose of encountering a distant comet, orbiting it and monitoring its activity along the comet's orbit as it approaches the Sun. The Rosetta spacecraft will encounter comet 64/P Churyumov Gerasimenko early 2014, will deliver a landing module in November 2014, and monitor the evolution of its activity until perihelion in August 2015. Within the scientific payload, the Microwave Instrument for the Rosetta Orbiter (MIRO) consists of a $30-\mathrm{cm}$ parabolic antenna equipped with two heterodyne receivers operating in the vicinity of two strong water vapor transitions, at $183 \mathrm{GHz}$ and at $557 \mathrm{GHz}$. In addition, two broadband channels operate around 562 and $190 \mathrm{GHz}$. The sub-millimeter receiver includes a high-resolution spectrometer tuned to measure four cometary gases $\left(\mathrm{H}_{2}^{16} \mathrm{O}, \mathrm{CO}\right.$, $\mathrm{NH}_{3}$, and $\left.\mathrm{CH}_{3} \mathrm{OH}\right)$ and two water isotopes $\left(\mathrm{H}_{2}^{17} \mathrm{O}\right.$ and $\left.\mathrm{H}_{2}^{18} \mathrm{O}\right)$. These data will be used to measure the surface and subsurface temperature, the gas production rates and relative abundances, the

\footnotetext{
* Corresponding author. Tel.: +33 140512012.

E-mail address: carlos.jimenez@obspm.fr (C. Jiménez).
}

velocity of each species, and to monitor these quantities as a function of location and heliocentric distance.

In order to reach the comet, the Rosetta spacecraft needed gravitational assistance. This was achieved by three flybys of the Earth (March 2005, November 2007 and 2009). In addition to the flybys, Rosetta encountered two asteroids for scientific investigation, Steins in September 2008 and Lutetia in July 2010. Data recorded during the asteroid flybys have been used to determine the physical properties of their surfaces and to provide an upper limit of their water production rate (Gulkis et al., 2010, 2012). There was also one flyby of Mars (February 2007), but due to limited resources, no data were recorded by the MIRO instrument.

Although the main goal of the Earth swing-bys was to modify the spacecraft trajectory, they also provided an opportunity to check the MIRO instrument. Large sets of atmospheric data have been acquired by Earth-orbiting instruments; some of these instruments operate in the same spectral region as MIRO. For instance, extensive analyses have been performed at $557 \mathrm{GHz}$ by the Odin satellite using limb sounding (e.g., Urban et al., 2007). The Aura EOS-MLS instrument has made similar measurements in the $183 \mathrm{GHz}$ water vapor transition, as well as at sub-mm frequencies (e.g., around $640 \mathrm{GHz}$ ) to derive the composition of minor chemical species (Waters et al., 2006). This wealth of data means that expected observational results at MIRO channels can 
be simulated with a large degree of confidence, which allows to check the consistency and quality of the MIRO data. Notice that, as far as we know, the MIRO data appear to be the only submillimeter high-resolution sounding of atmospheric $\mathrm{H}_{2} \mathrm{O}$ in the nadir mode.

The spectrum of the Earth in the $535-580 \mathrm{GHz}$ range, as viewed from space, is dominated by the strong $\mathrm{H}_{2}^{16} \mathrm{O}$ ground state rotational water vapor line centered at $556.936 \mathrm{GHz}$. Its complex shape is a tracer of the thermal vertical profile and opacity profile throughout the atmosphere. Fig. 1 shows a set of temperature and water vapor vertical profiles inferred from Earth-orbit observations for different seasons and latitudes, together with the corresponding simulated spectra around the $557 \mathrm{GHz}$ line observed at nadir. Beyond $2 \mathrm{GHz}$ from the line center, the far wings probe the troposphere. The minimum temperature which appears at about $2 \mathrm{GHz}$ from the center probes the temperature at the tropopause (between 10 and $15 \mathrm{~km}$ ). The maximum temperature observed at $10-20 \mathrm{MHz}$ from the line center probes the second maximum at about $50 \mathrm{~km}$ (stratopause). The central core, observed on the high-resolution spectrum, probes the mesosphere up to $80-85 \mathrm{~km}$. Several minor species can be identified in the wings of the strong $556.9 \mathrm{GHz}$ water vapor line, including the $\mathrm{H}_{2}^{18} \mathrm{O}$ transition at $547.6 \mathrm{GHz}$, also detected in the MIRO data. The changes in shape, both in the far wings and in the central core, are related to the different atmospheric conditions, and illustrate the potential of observations around this line to probe the terrestrial atmosphere. Notice that observations of Earth at submillimeter frequencies generally are sensitive not only to trace
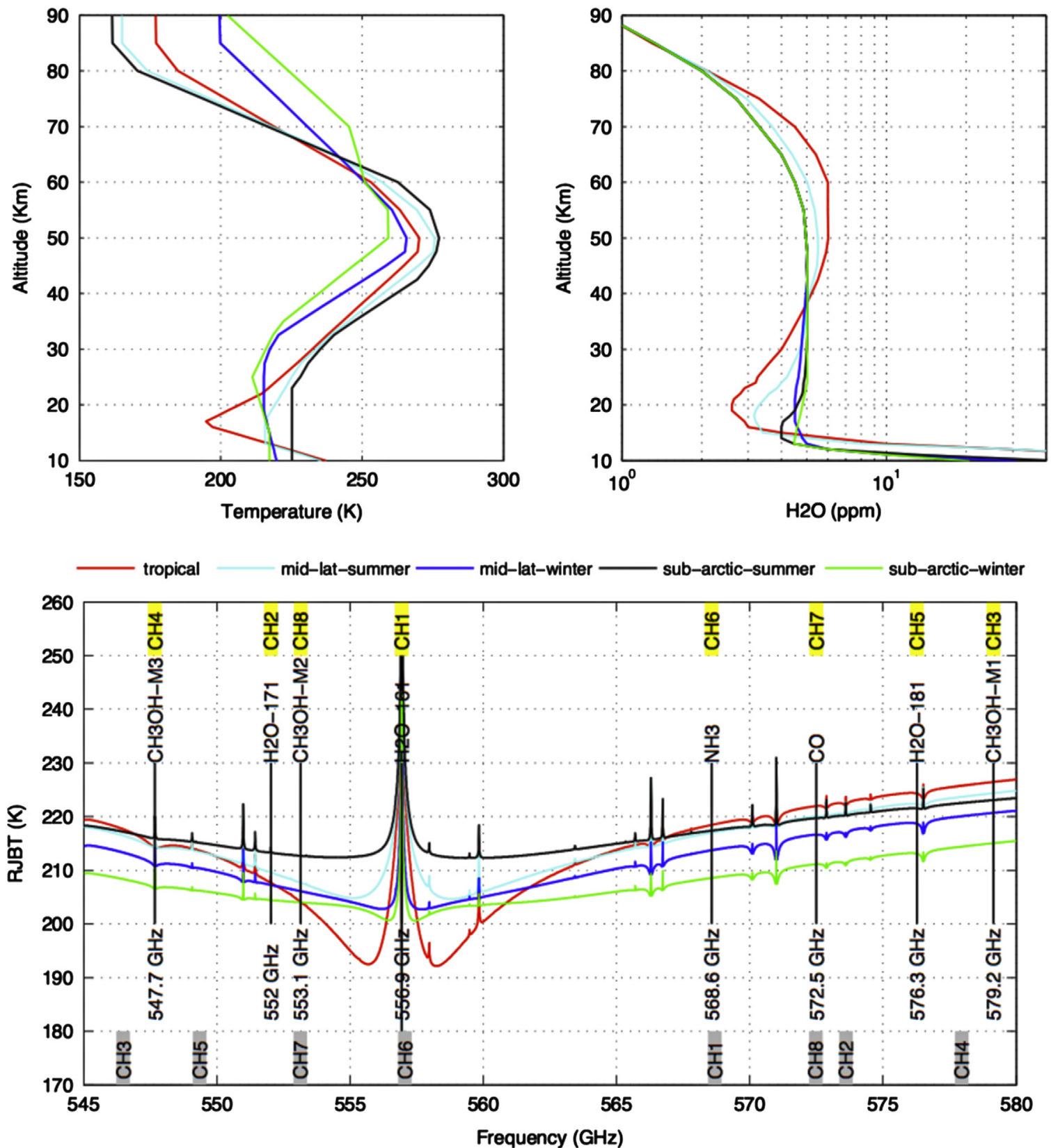

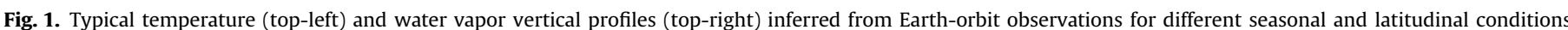

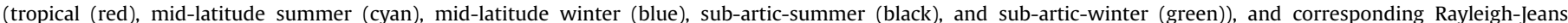

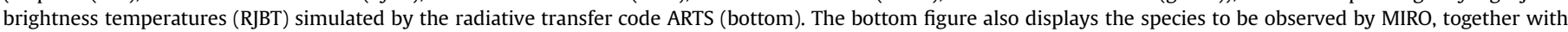

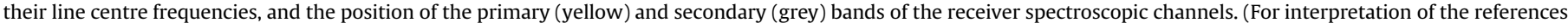
to color in this figure caption, the reader is referred to the web version of this article.) 
gases, but also to cloud ice particles (Jiménez et al., 2007). However, this can be ignored here, since we are focusing on the upper parts of the atmosphere.

The present paper describes the analysis of the MIRO data recorded during the second Earth flyby in November 2007. Section 2 describes the instrument. The inversion methodology is discussed in Section 3. Section 4 presents the MIRO observations, the expected observational results, and a technical discussion of the observed differences. The retrieval of the temperature profile from the MIRO observations is detailed in Section 5. Finally, the conclusions are summarized in Section 6.

\section{The MIRO instrument}

The MIRO instrument consists of a telescope equipped with two heterodyne receivers. A full description can be found in Gulkis et al. (2007). The primary mirror of the telescope is a $30 \mathrm{~cm}$ diameter offset parabolic antenna. The secondary mirror is an hyperboloid subreflector designed to provide very low sidelobes. The optical system, including a polarization grid as a frequency splitter, is designed to minimize alignment sensitivity to the large temperature range expected in the environment during the different phases of the mission (typically $100 \mathrm{~K}-300 \mathrm{~K}$ ). The instrument has a total mass close to $20 \mathrm{~kg}$ and operates over a power range of 19-72 W.

The two heterodyne receivers operate at millimeter wavelengths (190 GHz, i.e., $1.6 \mathrm{~mm}$ ) and sub-millimeter wavelengths ( $562 \mathrm{GHz}$, i.e., $0.5 \mathrm{~mm}$ ), respectively. At $190 \mathrm{GHz}$, a single broadband continuum detector is designed for the determination of the brightness temperature of comet $64 \mathrm{P} / \mathrm{CG}$ and the asteroids observed during flybys. At $562 \mathrm{GHz}$, in addition to a broadband receiver, a high-resolution spectrometer allows the simultaneous observation of 8 Double Side Band (DSB) channels centered on the specific transitions of cometary gaseous species. The bandwidth of each channel is $20 \mathrm{MHz}$ and the spectral resolution is $44 \mathrm{kHz}$, corresponding to a resolving power of about $10^{7}$. The molecular transitions are described in Table 1 , together with their line frequencies, the frequency position of the line in the receiver secondary band, and the assigned receiver channel number. The bandwidth of the spectral channel will allow observations over Doppler shifts of $5.4 \mathrm{~km} / \mathrm{s}$ or $8 \mathrm{~km} / \mathrm{s}$ with frequency switching. This will allow short spectral observations of the asteroids near closest approach, and measurements of low velocity molecular clouds. The very high spectral resolution is required to measure the line profiles of the cometary species which are Doppler

Table 1

Description of the molecular transitions observed by MIRO, together with the band numbering of the MIRO double side band sub-mm receiver and the frequency center of the image bands. The frequencies are given in $\mathrm{GHz}$. The sub-mm receiver also operates a continuum channel at $569.813 \mathrm{GHz}$ with the image band at $555.813 \mathrm{GHz}$

\begin{tabular}{lllll}
\hline Species & $\begin{array}{l}\text { Rotational } \\
\text { transition }\end{array}$ & $\begin{array}{l}\text { Line centre } \\
\text { frequency }\end{array}$ & $\begin{array}{l}\text { Side band centre } \\
\text { frequency }\end{array}$ & $\begin{array}{l}\text { Receiver } \\
\text { channel } \\
\text { number }\end{array}$ \\
\hline $\mathrm{H}_{2}^{16} \mathrm{O}$ & $1(1,0)-1(0,1)$ & 556.936 & 568.690 & 0 \\
$\mathrm{H}_{2}^{17} \mathrm{O}$ & $1(1,0)-1(0,1)$ & 552.020 & 573.604 & 1 \\
$\mathrm{CH}_{3} \mathrm{OH}(\mathrm{M} 3)$ & $12(-1)-11$ & 579.151 & 546.475 & 2 \\
& $(-1) \mathrm{E}$ & & & \\
$\mathrm{H}_{2}^{18} \mathrm{O}$ & $1(1,0)-1(0,1)$ & 547.676 & 577.950 & 3 \\
$\mathrm{CO}$ & $\mathrm{J}(5-4)$ & 576.267 & 549.358 & 4 \\
$\mathrm{CH} \mathrm{H}_{3} \mathrm{OH}(\mathrm{M} 2)$ & $3(-2)-2(-1)$ & 568.566 & 557.060 & 5 \\
& $\mathrm{E}$ & & & \\
$\mathrm{NH}_{3}$ & $\mathrm{~J}(1-0)$ & 572.498 & 553.128 & 6 \\
$\mathrm{CH}_{3} \mathrm{OH}(\mathrm{M} 1)$ & $8(1)-7(0) \mathrm{E}$ & 553.146 & 572.480 & 7 \\
\hline
\end{tabular}

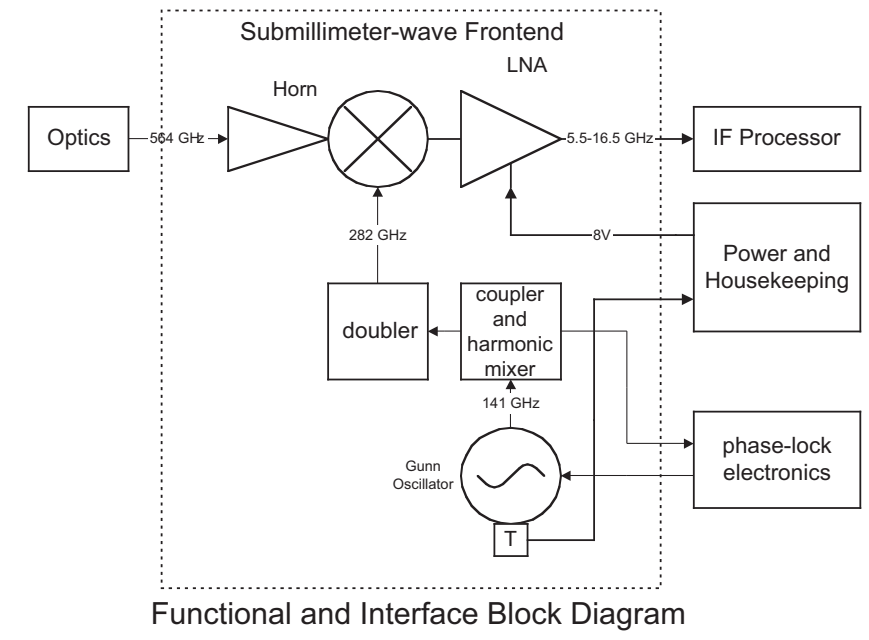

Fig. 2. Schematic diagram of the MIRO sub-millimeter heterodyne receiver (from Gulkis et al., 2007).

broadened. For the water transition at $557 \mathrm{GHz}$, at $10 \mathrm{~K}$, the full width at half-maximum of the Doppler-broadened line is $296 \mathrm{kHz}$. A resolving power higher than $2 \times 10^{6}$ is thus required to resolve the line.

The sub-millimeter receiver operates both as a single broadband radiometer for continuum measurements, and as a spectroscopic receiver. The front end (Fig. 2) consists of a feed horn, a subharmonically pumped (SHP) mixer, a low-noise amplifier, a frequency doubler and a local oscillator. The DSB receiver is fixed tuned covering the $552-579.5 \mathrm{GHz}$ frequency range to observe the MIRO target molecules, and uses a local oscillator (LO) frequency of $282 \mathrm{GHz}$ (half the signal frequency) to down convert the signal frequency to the range $5.5-16.5 \mathrm{GHz}$, where the signal is amplified by a Low-Noise Amplifier (LNA) and fed to an Intermediate Frequency Processor (IFP). The SHP mixer uses a pair of GaAs Shottky diodes. The LO signal is provided by a Gunn oscillator phase-locked from a ultra high stability frequency reference oscillator (USO).

The phase-locked Gunn oscillator is designed to shift its frequency rapidly by $\pm 15 \mathrm{MHz}$ from the center frequency thereby causing any observed spectral lines to shift their positions symmetrically by $\pm 15 \mathrm{MHz}$ on either side of the nominal line frequency. The frequency is alternately switched $\pm 15 \mathrm{MHz}$ every $5 \mathrm{~s}$. A frequency switched spectral line is spaced $10 \mathrm{MHz}$ apart in a differential spectrum constructed by subtracting ( $5 \mathrm{~s}$ ) spectra made during the alternating $10 \mathrm{~s}$ switch cycle time. This mode of operation is known as frequency switching. The receiver can also be operated in a second mode, referred to as asteroid mode. Because encounter velocities are large, a given spectral line moves quickly through the MIRO passband as a result of the Doppler shift. In asteroid mode, the frequency switching is turned off, and the first local oscillator is offset by $5 \mathrm{MHz}$ (plus or minus depending on the line under consideration) until closest approach. The LO is then switched in the opposite direction. This has the effect of increasing the effective passband of the spectrometer by $10 \mathrm{MHz}$.

The IFP is needed to process the IF output signal from the two front-ends. A schematic drawing of the IFP is shown in Fig. 3. The continuum channels are shown at the top of the figure above the dashed line. The spectroscopic part of the IFP is shown below the dashed line. The submm wave signal is down converted by the SHP mixer to a first IF band of $5.5-16.5 \mathrm{GHz}$. Table 2 lists the observed lines along with the various frequencies associated with the IFP inside this range. A divider separates out the continuum band while the spectroscopic signal is further down converted for input to the spectrometer (frequency range of $180 \mathrm{MHz}$ centered at 


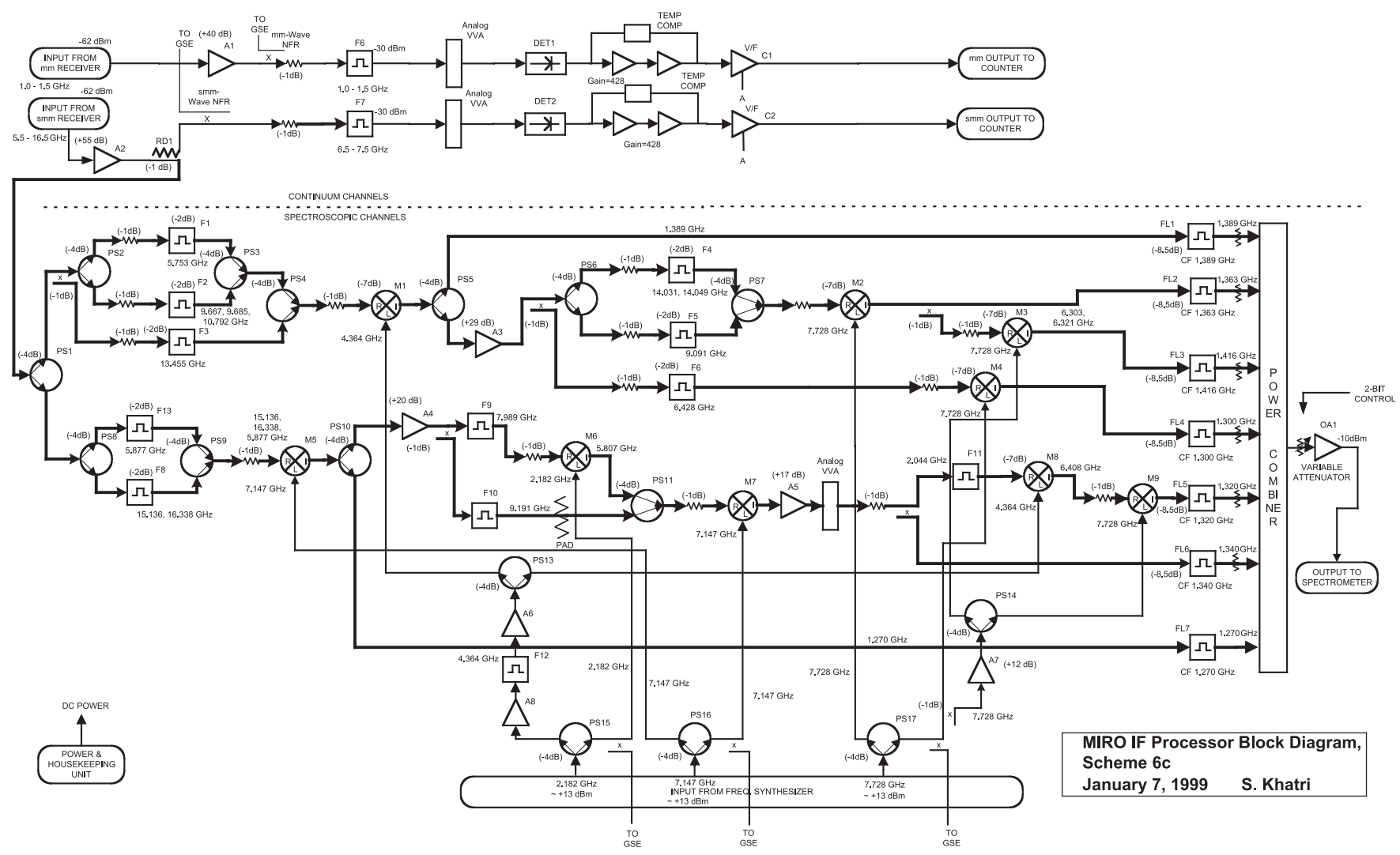

Fig. 3. Schematic drawing of the Intermediate Frequency Processor (IFP) (from Gulkis et al., 2007).

Table 2

Summary of the down converted frequencies for each spectral line of the down converted spectrum for input into the spectrometer (all frequencies are given in MHz). The last column gives the IFP output filter used for each channel before the CTS.

\begin{tabular}{|c|c|c|c|c|c|c|c|c|c|c|c|c|}
\hline Channel LO & $\mathrm{RF}$ & IF 1 & $\begin{array}{l}\text { IF } 2 \\
\text { M1 out } \\
2 \times 2182\end{array}$ & $\begin{array}{l}\text { IF } 3 \\
\text { M2 out } \\
7728\end{array}$ & $\begin{array}{l}\text { IF } 4 \\
\text { M3 out } \\
7728\end{array}$ & $\begin{array}{l}\text { IF } 5 \\
\text { M4 out } \\
7147\end{array}$ & $\begin{array}{l}\text { IF } 6 \\
\text { M5 out } \\
2182\end{array}$ & $\begin{array}{l}\text { IF } 7 \\
\text { M6 out } \\
7147\end{array}$ & $\begin{array}{l}\text { IF } 8 \\
\text { M7 out } \\
2 \times 2182\end{array}$ & $\begin{array}{l}\text { IF 9 } \\
\text { M3 out } \\
7728\end{array}$ & Output to CTS & Filter to CTS \\
\hline 0 & 556936 & 5877 & - & - & - & 1270 & - & - & - & - & 1270 & FL7 \\
\hline 2 & 579151 & 16338 & - & - & - & 9191 & - & 2044 & 6408 & 1320 & 1320 & FL5 \\
\hline 3 & 547677 & 15136 & - & - & - & - & 7989 & 5807 & 1340 & - & 1340 & FL6 \\
\hline 4 & 576268 & 13455 & 9091 & 1363 & - & - & - & - & - & - & 1363 & FL2 \\
\hline
\end{tabular}

$1.350 \mathrm{GHz}$ ). The IFP uses 3 oscillators along with 9 mixers (circles with crosses) and filters to produce 7 output bands (the LOs are used multiple times to save power). Nominally $20 \mathrm{MHz}$ wide filters are used in the IFP before input to the spectrometer to eliminate excess noise but large enough for including the Doppler shifts. The output filters are indicated as FL1-FL7 on the right side of the figure. All filters contains one spectral line, apart from FL3, that contains 2 spectral lines. The seven filters are on the right-hand side of the figure, just before the Power Combiner. The IFP output filter used for each channel before the CTS is given in Table 2.

The high spectral resolution $\left(10^{7}\right)$ is achieved by a 4096 channel Chirp Transform Spectrometer (CTS, Hartogh and Hartmann , 1990). The CTS is used to measure in real time the power spectrum of the 8 channels on 7 output bands of frequencies from the IFP with a resolution of $44 \mathrm{KHz}$ each channel. The spectrometer output is a digitized power spectrum supplied to the Command and Data Handling System.

\section{Inversion methodology}

\subsection{The inversion problem}

Following the formalism of Rodgers (1990), the observation forward model, F, can be linearized as

$\mathbf{y}=\mathrm{F}\left(\mathbf{x}_{a}, \mathbf{b}_{a}\right)+\mathbf{K}_{\mathbf{x}}\left(\mathbf{x}-\mathbf{x}_{a}\right)+\mathbf{K}_{\mathbf{b}}\left(\mathbf{b}-\mathbf{b}_{a}\right)+\epsilon$,

where $\mathbf{y}$ is the radiance vector, the vector $\mathbf{x}$ groups the parameters to be retrieved, the vector $\mathbf{b}$ contains the remaining parameters (defining the atmospheric state, radiative transfer calculations, and the sensor characteristics), $\mathbf{x}_{a}$ and $\mathbf{b}_{a}$ are respectively the best a priori knowledge of the state and forward model parameters, $\mathbf{K}_{\mathbf{x}}=\partial \mathbf{y} / \partial \mathbf{x}$ and $\mathbf{K}_{\mathbf{b}}=\partial \mathbf{F} / \partial \mathbf{b}$ are respectively the state and forward model parameter weighting functions, and $\epsilon$ refers to measurement errors not covered by b (typically the spectral thermal noise). 
The difference between retrieved, $\hat{\mathbf{x}}$, and true, $\mathbf{x}$, parameters is called the retrieval error, $\delta$, which can be expressed as

$\delta=\hat{\mathbf{x}}-\mathbf{X}=(\mathbf{A}-\mathbf{I})\left(\mathbf{X}-\mathbf{X}_{a}\right)+\mathbf{D}_{\mathbf{y}} \mathbf{K}_{\mathbf{b}}\left(\mathbf{b}-\mathbf{b}_{a}\right)+\mathbf{D}_{\mathbf{y} \epsilon}$,

where $\mathbf{A}=\partial \hat{\mathbf{x}} / \partial \mathbf{x}=\mathbf{D}_{\mathbf{y}} \mathbf{K}_{\mathbf{x}}$ is the averaging kernels, and $\mathbf{I}$ represents the identity matrix. $(\mathbf{A}-\mathbf{I})\left(\mathbf{x}-\mathbf{x}_{a}\right)$, is called the smoothing error and originates as not all features of the true state can be resolved due to the always limited vertical resolution of the observations. $\mathbf{D}_{\mathbf{y}} \epsilon$, and $\mathbf{D}_{\mathbf{y}} \mathbf{K}_{\mathbf{b}}\left(\mathbf{b}-\mathbf{b}_{a}\right)$ are called the measurement and forward model errors, respectively. They can be grouped together as $\mathbf{D}_{\mathbf{y}} \mathbf{e}$, where $\mathbf{e}=\mathbf{K}_{\mathbf{b}}\left(\mathbf{b}-\mathbf{b}_{a}\right)+\epsilon$ is called the observation uncertainty.

The exact value of the error cannot be calculated because obviously neither $\mathbf{x}$ nor $\mathbf{b}$ will ever be known, but the observation uncertainty can be characterized as

$\mathbf{S}_{\mathbf{e}}=\mathbf{K}_{\mathbf{b}} \mathbf{S}_{\mathbf{b}} \mathbf{K}_{\mathbf{b}}^{T}+\mathbf{S}_{\epsilon}$

where $\mathbf{S}_{\mathbf{b}}$ and $\mathbf{S}_{\epsilon}$ are the covariance matrices expressing the measurement and forward model parameters uncertainty. The retrieval error $\mathbf{S}_{\delta}$ can then be estimated as

$\mathbf{S}_{\delta}=\mathbf{S}_{\mathbf{m}}+\mathbf{S}_{\mathbf{o}}=(\mathbf{A}-\mathbf{I}) \mathbf{S}_{\mathbf{x}}(\mathbf{A}-\mathbf{I})^{T}+\mathbf{D}_{\mathbf{y}} \mathbf{S}_{\mathbf{e}} \mathbf{D}_{\mathbf{y}}^{T}=\mathbf{K}_{\mathbf{x}}^{T} \mathbf{S}_{\mathbf{e}}^{-1} \mathbf{K}_{\mathbf{x}}+\mathbf{S}_{\mathbf{x}}^{-1}$,

where $\mathbf{S}_{\mathbf{m}}$ and $\mathbf{S}_{\mathbf{o}}$ are the contributions of the smoothing and observation errors, respectively, to the statistical description of the retrieval error, and $\mathbf{S}_{\mathbf{x}}$ is the covariance matrix characterizing the uncertainty in the retrieved parameters.

\subsection{The inverse model}

Concrete expressions for the retrieved parameters $\hat{\mathbf{x}}$ and retrieval error $\mathbf{S}_{\delta}$ can only be derived by selecting a specific inverse model. A possibility is to derive first the a posteriori probability distribution function of the state to be retrieved given the measurement, and then selecting a retrieved state by maximising the a posteriori pdf. This is the so-called maximum a posteriori solution. If Gaussian statistics are assumed, maximising the a posteriori distribution is equivalent to minimising the function

$\mathbf{f}(\mathbf{x})=\left[\mathbf{y}-\mathrm{F}\left(\mathbf{x}, \mathbf{b}_{a}\right)\right]^{T} \mathbf{S}_{\mathbf{e}}^{-1}\left[\mathbf{y}-\mathrm{F}\left(\mathbf{x}, \mathbf{b}_{a}\right)\right]+\left(\mathbf{x}-\mathbf{x}_{a}\right)^{T} \mathbf{S}_{\mathbf{x}}^{-1}\left(\mathbf{x}-\mathbf{x}_{a}\right)$,

expression commonly referred as the optimal estimation solution in the atmospheric literature (Rodgers, 1976). It can be observed that this expression is a form of regularization with a trade-off parameter of statistical nature weighting the measurement uncertainty and the apriori knowledge of the parameter to be estimated. If the expression is minimized the solution is given by the implicit equation for $\hat{\mathbf{x}}$

$\hat{\mathbf{x}}=\mathbf{x}_{a}+\mathbf{S}_{\mathbf{x}} \mathbf{K}_{\mathbf{x}}^{T} \mathbf{S}_{\mathbf{e}}^{-1}\left[\mathbf{y}-\mathrm{F}\left(\hat{\mathbf{x}}, \mathbf{b}_{a}\right)\right]$,

with the state weighting function evaluated at $\left(\hat{\mathbf{x}}, \mathbf{b}_{a}\right)$.

For non-linear inversion problems (as it is the case here), Eq. (6) has to be solved numerically. Gradient descent techniques are typically used. Here we will use the Marquardt-Levenberg algorithm

$\hat{\mathbf{x}}_{i+1}=\hat{\mathbf{x}}_{i}-\left(\mathbf{H}_{i}+\gamma \mathbf{I}\right)^{-1} \mathbf{g}_{i}$,

where $\mathbf{H}_{i}$ is the Hessian matrix, second derivatives of $\mathbf{f}(\mathbf{x})$ evaluated at $\hat{\mathbf{x}}_{i}, \gamma$ is a parameter controlling a trade-off between a steepest descent (first iterations) and a Newtonian descent with faster convergence (when approaching the minimum), and $\mathbf{g}_{i}$ is the first derivatives of $\mathbf{f}(\mathbf{x})$ evaluated at $\hat{\mathbf{x}}_{i}$ (Marks and Rodgers, 1993). By calculating the first and second derivatives of $\mathbf{f}(\mathbf{x})$, a step of the Marquardt-Levenberg iteration becomes

$\hat{\mathbf{x}}_{i+1}=\hat{\mathbf{x}}_{i}+\left(\mathbf{S}_{\mathbf{x}}^{-1}+\mathbf{K}_{i}^{T} \mathbf{S}_{\mathbf{e}}^{-1} \mathbf{K}_{i}+\gamma \mathbf{S}_{\mathbf{x}}^{-1}\right)^{-1}\left\{\mathbf{K}_{i}^{T} \mathbf{S}_{\mathbf{e}}^{-1}\left[\mathbf{y}-\mathrm{F}\left(\hat{\mathbf{x}}_{i}\right)\right]+\mathbf{S}_{\mathbf{x}}^{-1}\left[\mathbf{x}_{a}-\hat{\mathbf{x}}_{i}\right]\right\}$,

where $\mathbf{K}_{i}$ is the weighting functions matrix re-evaluated at each iterative state. Notice that $\gamma \mathbf{S}_{\mathbf{x}}^{-1}$ is preferred here to the more usual $\gamma \mathbf{I}$
Table 3

Summary of MIRO Earth swing-bys data acquisitions. CA stands for closest approach.

\begin{tabular}{llll}
\hline Reference & $\begin{array}{l}\text { Time period } \\
(\mathbf{k m})\end{array}$ & $\begin{array}{l}\text { Closest } \\
\text { distance }\end{array}$ & Operation mode \\
\hline ESB1 & $\begin{array}{l}2005 / 03 / 04 \\
\text { T08:09 }\end{array}$ & 8331 & Asteroid mode near CA \\
& $\begin{array}{l}\text { 2005/03/07 } \\
\text { T14:02 }\end{array}$ & & $\begin{array}{l}\text { Frequency switching for the } \\
\text { rest }\end{array}$ \\
& $2007 / 11 / 13$ & 11,647 & Asteroid mode near CA \\
ESB2 & T12:09 & & $\begin{array}{l}\text { Frequency switching for the } \\
\text { rest }\end{array}$ \\
& $2007 / 11 / 20$ & & Frequency switching \\
& T12:09 & & \\
ESB3 & $2009 / 11 / 07$ & 8858 & \\
& T21:00 & & \\
& $2009 / 11 / 18$ & & \\
& T07:40 & &
\end{tabular}

from Eq. (7) to take into account the possible large range of values in the state vector (e.g., if species with very different absolute values are retrieved at the same time).

Apart from the iterative method, criteria to halt the iteration is also needed. Here we will look at the difference of $\hat{\mathbf{x}}_{i+1}-\hat{\mathbf{x}}_{i}$. As Gaussian statistics are assumed for the state vector, the variable $(\hat{\mathbf{x}}-\mathbf{x})^{T} \mathbf{S}_{\delta}^{-1}(\hat{\mathbf{x}}-\mathbf{x})$ follows a $\chi^{2}$ distribution of expected value $n$, where $n$ is the length of the state vector. Close to the solution the difference $\hat{\mathbf{x}}_{i+1}-\hat{\mathbf{x}}_{i}$ should be much smaller than $\hat{\mathbf{x}}-\mathbf{x}$, so the expected value of $\left(\hat{\mathbf{x}}_{i+1}-\hat{\mathbf{x}}_{i}\right)^{T} \mathbf{S}_{\delta}^{-1}\left(\hat{\mathbf{x}}_{i+1}-\hat{\mathbf{x}}_{i}\right)$ should also be much smaller than $n$. Consequently a reasonable test for convergence is

$\left(\hat{\mathbf{x}}_{i+1}-\hat{\mathbf{x}}_{i}\right)^{T} \mathbf{S}_{\delta}^{-1}\left(\hat{\mathbf{x}}_{i+1}-\hat{\mathbf{x}}_{i}\right) \ll n$,

stopping the iterations when, for instance, the above expression becomes one order of magnitude smaller than $n$.

\subsection{Practical implementation}

The Atmosphere Radiative Transfer Simulator (ARTS) (Buehler et al., 2004; Eriksson et al., 2011) is used here as the forward model introduced in Section 3.1. ARTS is a general software package for long wavelength radiative transfer simulations, including a rigorous treatment of scattering and full description of the polarization state (Davis et al., 2007), and intended to be as general as possible in order to handle most of the existing Earth observation geometries from different ground, airborne, and satellite platforms. To support remote sensing applications, weighting functions can be calculated for the most important atmospheric variables in non-scattering conditions, and a comprehensive and efficient treatment of sensor characteristics is included (Eriksson et al., 2006). ARTS is well validated by intercomparison studies with other radiative transfer models (Buehler et al., 2006; Melsheimer et al., 2005) and has been used in a range of different research areas related to thermal radiation in the Earth's atmosphere. In the context of an ongoing study financed by the European Space Agency, ARTS is currently being extended to handle atmospheres of other planets, specifically Mars, Venus, and Jupiter, besides Earth.

The choice of spectroscopic parameters can have some influence on retrieval results (e.g., Verdes et al., 2005). ARTS can use some specialized spectral line catalogues, such as Perrin et al. (2005), but for this study we used the HITRAN 2008 catalogue (Rothman et al., 2009) for the molecular lines, and Rosenkranz (1993) for the modeling of the $\mathrm{H}_{2} \mathrm{O}, \mathrm{O}_{2}$, and $\mathrm{N}_{2}$ continuum absorption. ARTS uses a lookup table approach to pre-calculate gas absorption (Buehler et al., 2011). For broad-band calculations, 
this can be combined with an optimized method to select representative frequencies (Buehler et al., 2010) to speed up calculations even further. Different radiance units can be used in ARTS radiative transfer calculations (see, e.g., Eriksson et al., 2011).

ARTS is freely available, maintained as an open-source project, and comes with a collection of high level functions for interaction with ARTS. Part of this collection is a Matlab package called Qpack (Eriksson et al., 2004), dedicated to atmospheric retrieval work. Qpack is used here to practically implement the inverse model described in Section 3.2 and conduct the inversions of the MIRO observations. For more information about ARTS, related tools, and present range of applications, see www.sat.1tu.se/arts.

\section{The MIRO observations}

The details of the three Earth swing-bys and the MIRO operation mode are given in Table 3. ESB1 data appear to be of poor quality, probably because of the large Doppler shift; ESB3 data were taken in the frequency-switch mode so that the continuum was removed. Therefore, we choose the ESB2 data acquired in asteroid mode for a comparison of observed and expected atmospheric spectrum.

The spectra obtained during the ESB2 flyby in the asteroid mode for the 8 MIRO spectroscopic channels are shown in Fig. 4, together with some expected spectra from simulations with the radiative transfer code ARTS. Each spectrum has been averaged from the 112 individual spectra recorded during the Earth flyby 13 November 2007 between UTC 20:50-21:08. At that time Rosetta was overpassing the southern part of the Indian Ocean (from 62E$25 \mathrm{~S}$ to $57 \mathrm{E}-100 \mathrm{~N}$ ). Notice that MIRO radiances are reported as Rayleigh-Jeans brightness temperatures, defined as

$\operatorname{RJBT}(\nu)=\frac{c^{2}}{2 k_{B} \nu^{2}} I(\nu)$,

where $\nu$ is the frequency, $c$ is the speed of light, $k_{B}$ is the Boltzmann constant, and $I$ is the total power per unit frequency per unit area per solid angle measured by the instrument $\left(\mathrm{Wm}^{-2} \mathrm{~Hz}^{-1} \mathrm{Sr}^{-1}\right)$. This unit is
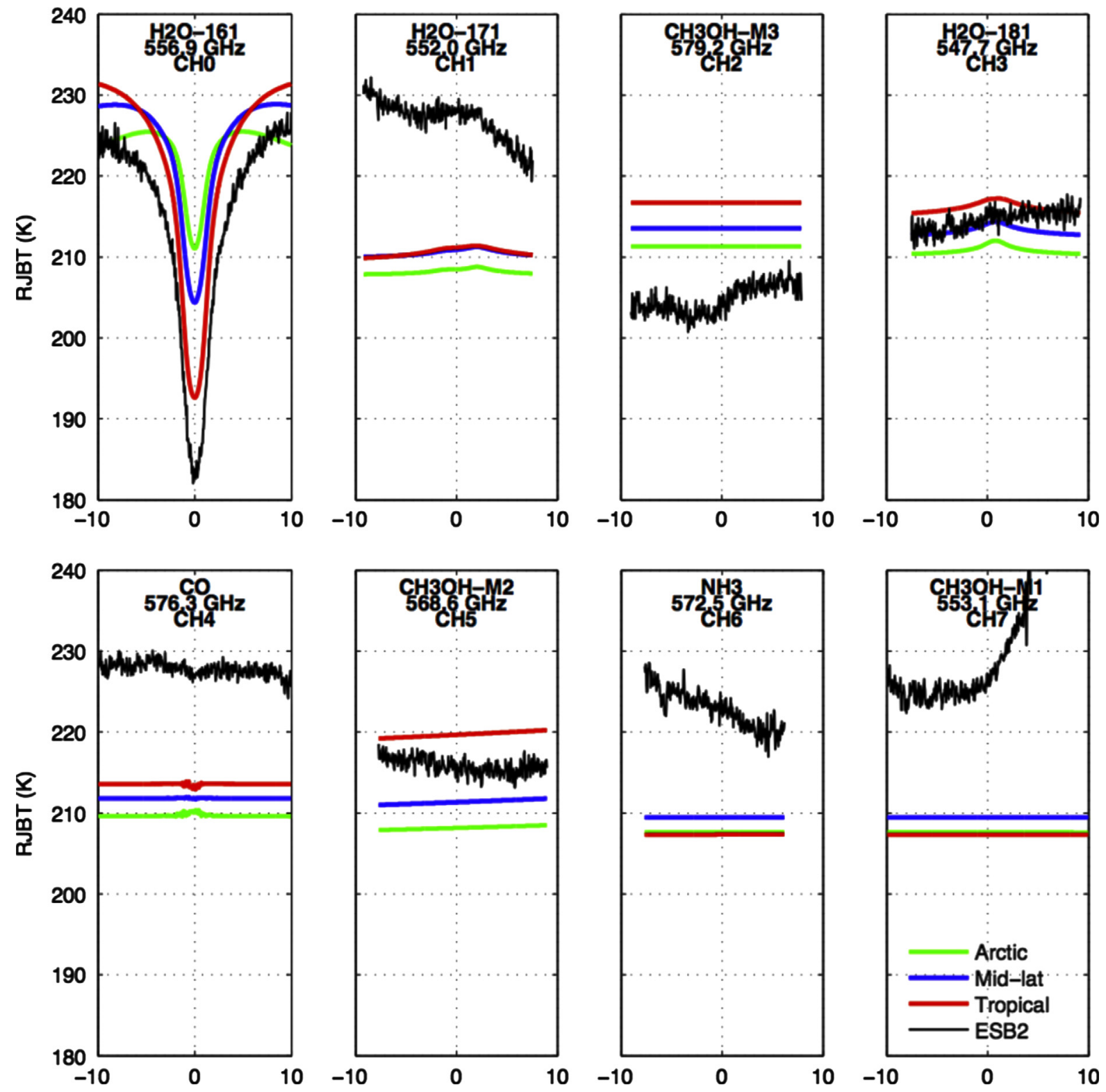

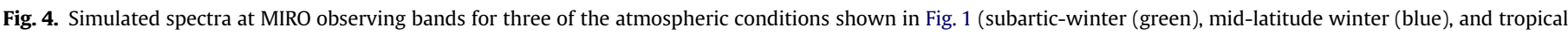

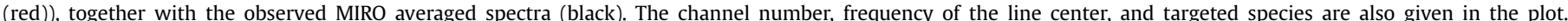
(For interpretation of the references to color in this figure caption, the reader is referred to the web version of this article.) 

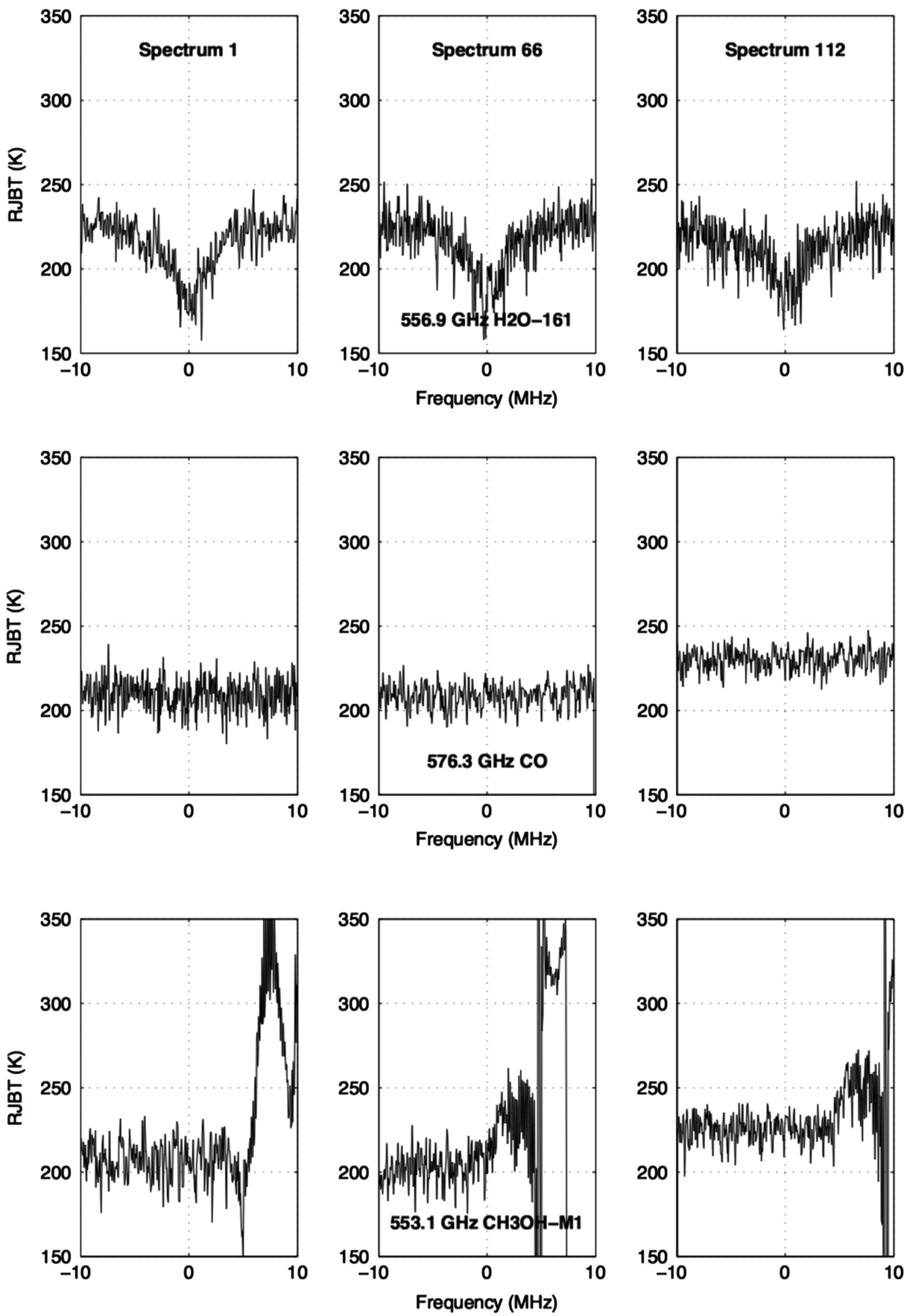

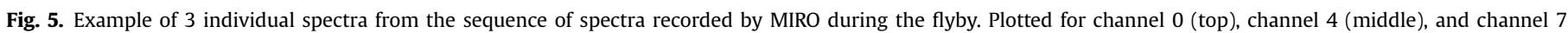
(bottom) the first (left), in the middle (middle), and last (right) spectrum in the sequence of 112 recorded spectra. 
derived from the Rayleigh-Jeans approximation of the Planck function describing the energy a blackbody emits. Notice also that at the MIRO sub-millimetre frequencies the Rayleigh-Jeans brightness temperature is lower than the physical temperature (of the order of $10 \mathrm{~K}$ for the range of radiances measured by MIRO).

Fig. 4 shows the $\mathrm{H}_{2}^{16} \mathrm{O}$ central core easily detected in absorption by MIRO. The simulated spectra corresponds to three of the atmospheres presented in Fig. 1 at nadir. Comparing the observed and the simulated averaged spectra shows large differences for some channels. For channels 1, 4, 6, and 7, the observed spectra are outside the range of expected brightness temperatures, and show spectral shapes that cannot be reproduced in the forward modeling of these bands. Notice for instance the nearly identical simulated radiances for channels 6 and 7 (expected as the lower and upper channel 6 sidebands fall onto the upper and lower channel 7 sidebands), but the different MIRO observed radiances. The channel 2 radiances are closer to the expected values, but the spectral shape is not as flat as the simulations suggest.

Inspection of the sequence of individually recorded spectra for the affected channels explained some of the observed spectral artifacts. Examples of individual spectra for channels 0, 4, and 7 are given in Fig. 5. For instance, for channel 4 the spectra 1 and 61 have brightness temperatures as expected (around $210 \mathrm{~K}$ ), but not the spectrum 112. These offsets observed in some of the spectra seem too large to be justified by changing temperature and water vapor conditions. In some occasions zero brightness temperatures and unrealistic brightness temperatures for these atmospheric observations (well above $300 \mathrm{~K}$ ) are reported by the instrument. Both are visible in the channel 7 spectra of Fig. 5.
The very large brightness temperatures only happened for channel 7 and are responsible for the unusual shape of its averaged spectrum at the end of the band.

These spectral artifacts suggested anomalies in the operations of the radiometer at the time of the EBS2 flyby, and prompted an investigation that confirmed that high level fluctuation anomalies can be detected on these channels. These four channels correspond to the FL4, FL2, and FL3 output filters of the IFP (see the IFP schematic in Fig. 3 and the down-converted frequencies in Table 2). Their filter outputs are located on the upper part (except for FL1) of the spectroscopic channel scheme of the IFP. This is consistent with the electronic parts in between the IFP IF power divider PS5 and the output power combiner. The more suspicious component common to the three fluctuating outputs is the A3 IF amplifier, which could be having higher noise or gain variations than expected.

Looking at the noise fluctuation on all channels in Fig. 4, large differences between the good and the anomalous channels are not observed. This has been confirmed by the analysis of Kamp (2012): the theoretical noise fluctuation for an average system temperature of $8000 \mathrm{~K}$ and a bandwidth of $44 \mathrm{kHz}$ for $5 \mathrm{~s}$ integration time is $13.9 \mathrm{~K}$, while the measured noise is in the $12.8-17.7 \mathrm{~K}$ range over all the spectral channels. This confirms that the anomalous bands are not significantly different from the good ones in either their mean band average or the spectral noise. In conclusion, the noise fluctuation values are in reasonable agreement with the expected standard deviation from the noise formula, $13.9 \mathrm{~K}$, and do not explain the bigger anomalies seen on the four channel observations. Therefore, there is a strong possibility that the anomalous
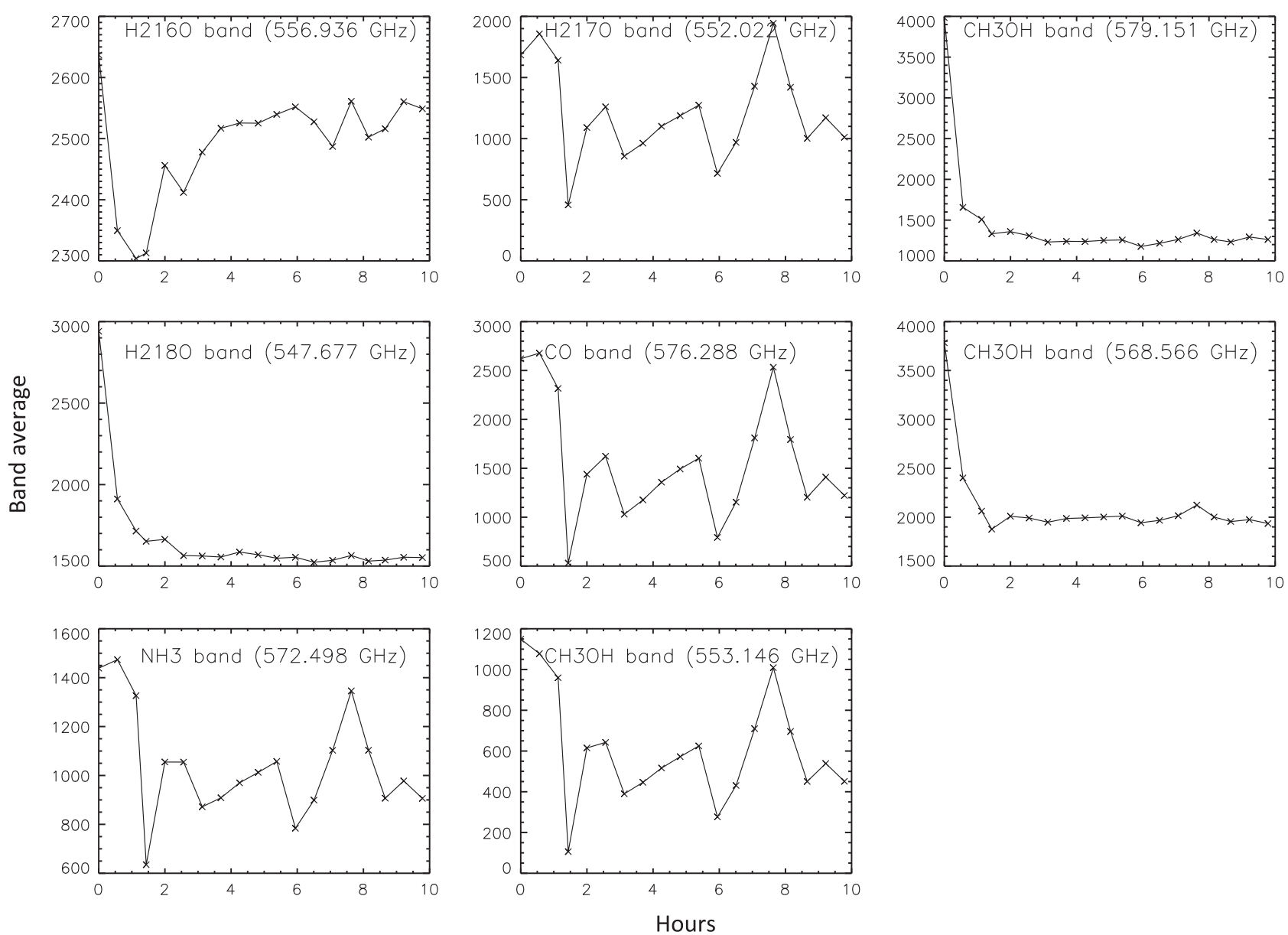

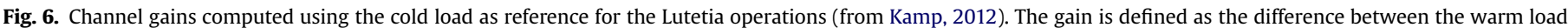
raw counts and those of the spectrum used as the cold reference, divided by the temperature difference. 

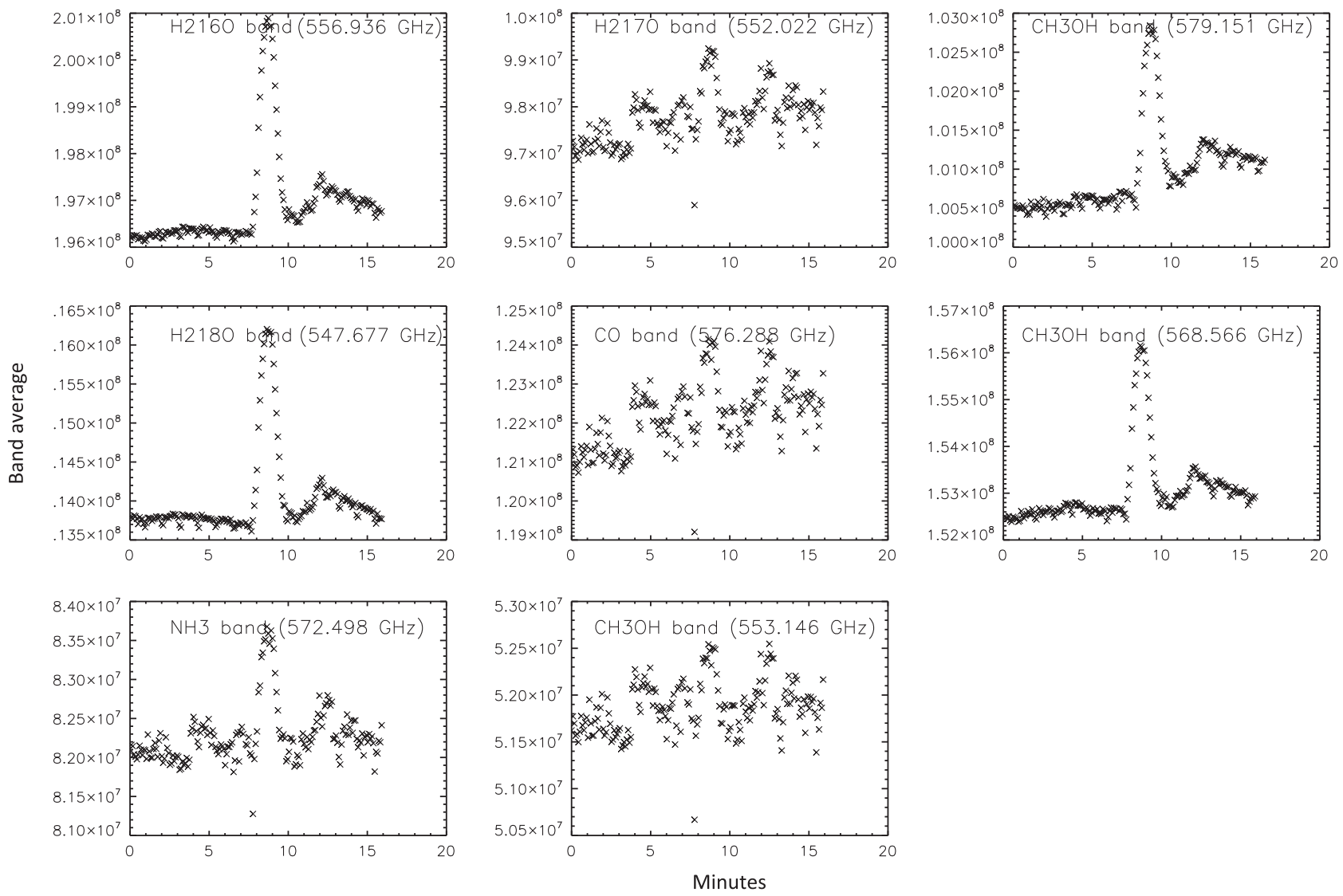

Fig. 7. Band averages (using the standard performance-monitoring bands) for the raw asteroid-mode CTS data for the Steins encounter (from Kamp, 2012).

behavior of these bands is due to gain instabilities in amplifier A3, which cause occasional large fluctuations in their signal.

Further instrumental tests are pointing out in this direction. For instance, the channel gains have been computed using the cold load during the Lutetia asteroid flyby (Gulkis et al., 2012) and are reported in Fig. 6. It can be seen that the averaged computed gains for the four anomalous channels are consistently lower than for the remaining ones, and that the behavior of the four anomalous bands is highly correlated. This is particularly noticeable in the data from the Lutetia flyby and seems to confirm that the A3 amplifier unstable gain is responsible for the anomalous behavior. The data from the Steins asteroid encounter (Gulkis et al., 2010) have also been analyzed. The band averages for this flyby are displayed in Fig. 7. It can be seen that the signal from the asteroid, which is clearly visible in the good bands, is also visible in the four anomalous ones, although in the latter it is obscured, probably by the likely amplifier unstabilities.

So far the technical investigations have concentrated in the A3 amplifier, as its anomalous behavior can explain a large part of the unexpected variations in the observed spectra. Nevertheless, some of the other artifact, such as the unrealistic brightness temperatures output by the instrument at some occasions, may originate from other components and further technical investigations are currently in progress.

\section{Inversions of MIRO observations}

\subsection{Inversion strategy}

The state weighting functions $\mathbf{K}_{\mathbf{x}}$ are first analyzed to see the potential of the spectroscopic MIRO channels to sound the atmosphere. Fig. 8 shows the weighting functions for the atmospheric temperature and the water vapor content at selected frequencies for the different channels. Compared with typical weighting functions from atmospheric nadir sounders, there are not optimal in several ways. For instance, they do not cover all altitudes (see the absence of channels with sensitivity to the thermal emission at altitudes around 25 and $40 \mathrm{~km}$ ). The DSB receiver also implies that all channels show sensitivity to the emission in the troposphere (at $\sim 10 \mathrm{~km}$ ) due to the contribution from the image band (see the image band center frequencies in Table 1). Although this can be useful in some cases (e.g., the weighting function for channel 5 adds some sensitivity at around $30 \mathrm{~km}$ due to an image band falling at $\sim 130 \mathrm{MHz}$ of the $557 \mathrm{GHz}$ line), in general this complicates the inversion problem due to the contribution of thermal emission from two different altitudes for each channel. Of course, it should be remembered that the placement of MIRO channels is optimized for cometary observations, not to sound the terrestrial atmosphere.

As the weighting function shows, the thermal emission from the $557 \mathrm{GHz}$ line at different altitudes depends both on the thermal profile and on the water vapor content. A retrieval of both profiles is in principle possible, but at the cost of a more uncertain inversion problem as the same information content is used to derive two quantities. In practice it is more common to retrieve the temperature from a dedicated observing instrumental channel (e.g., as in von Engeln et al., 1998 around the $60 \mathrm{GHz}$ $\mathrm{O}_{2}$ transition, where the $\mathrm{O}_{2}$ atmospheric composition is fixed and the observed spectra depends only on the thermal profile), and then using the retrieved temperature as input of an inversion solely dedicated to retrieve the atmospheric species of interest. Here the objective is to evaluate the observed MIRO spectra, rather than retrieving a specific atmospheric parameter, so we choose the following evaluation strategy: (1) to acquire a relatively accurate water vapor profile from an Earth observing instrument; (2) to use 

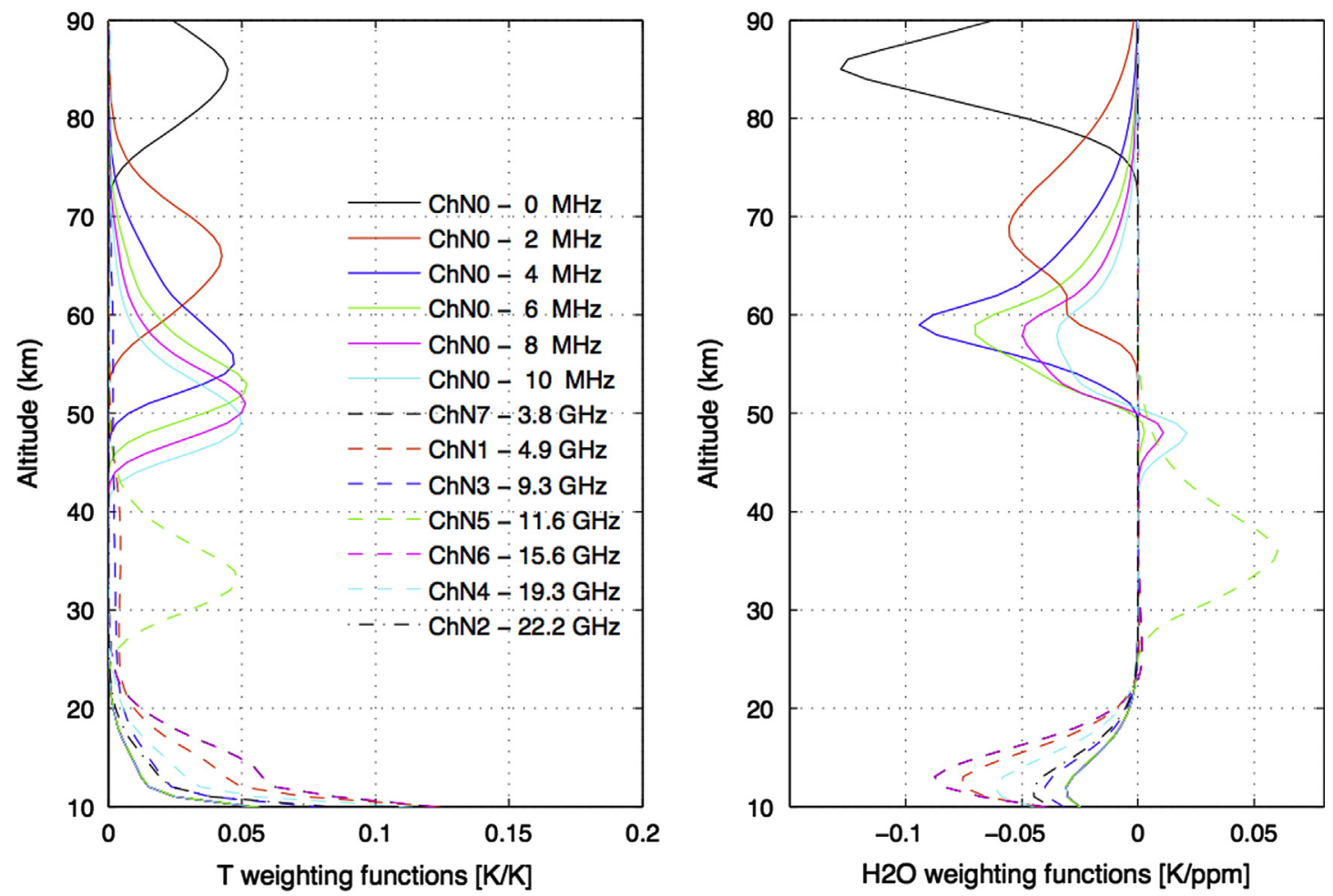

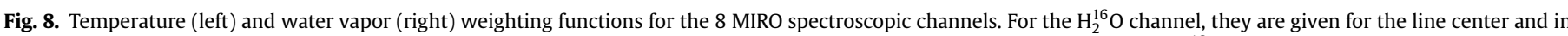

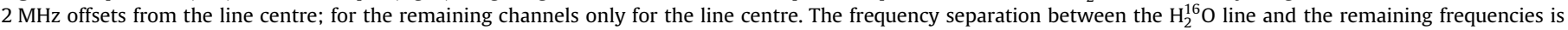
given together with the channel number.

the MIRO spectral information to retrieve the temperature profile, having the water vapor profile as input to the inversion, and; (3) to compare the retrieved temperature with the expected atmospheric temperature. The EOS-MLS microwave limb sounder (Waters et al., 2006) is selected here as the satellite instrument to observe the Earth atmosphere. EOS-MLS retrieves temperature from the $118 \mathrm{GHz}$ line, with precisions ranging from $\sim 1 \mathrm{~K}$ in the lower stratosphere to $\sim 2.5 \mathrm{~K}$ in the mesosphere (Schwartz and et al., 2008). The main water vapor product comes from inversions of the $183 \mathrm{GHz}$ line, with precisions of $\sim 0.2$ to $\sim 0.3 \mathrm{ppmv}$ in the stratosphere and $\sim 0.4$ to $\sim 1.1 \mathrm{ppmv}$ in the mesosphere (Lambert and et al., 2007). "Precision" here refers to the diagonal elements of the measurement error covariance matrix $\mathbf{S}_{\epsilon}$ (i.e., it mostly characterizes the propagation of radiometric noise into a retrieval error).

\subsection{Inversion of simulated spectra}

The observation system (i.e., the system formed by a concrete instrument and a specific inversion model) is tested first by inverting simulated spectra corresponding to the retrieved EOSMLS atmosphere for the day of the ESB2 flyby. The EOS-MLS atmosphere is constructed by averaging the profiles from overpasses close to the MIRO ground path during the day of the flyby. The retrieval is carried out by iteratively applying Eq. (8), as described in Section 3.2. The a priori temperature profile $\mathbf{x}_{a}$ is taken from the mid-latitude winter atmosphere shown in Fig. 1, and the temperature uncertainty setup as a covariance matrix $\mathbf{S}_{\mathbf{x}}$ with a standard deviation of $5 \mathrm{~K}$ and linear correlation lengths between $\sim 1.5 \mathrm{~km}$ (lower stratosphere) and $\sim 8.0 \mathrm{~km}$ (mesosphere) (see, e.g., Eriksson and Chen , 2002). The measurement error $\mathbf{S}_{\mathbf{e}}$ is setup as a diagonal matrix with a radiometric noise of $1 \mathrm{~K}$, to approximate the radiometric noise of the ESB2 averaged spectra. Fig. 9 shows the retrieved profiles when inverting all MIRO spectroscopic channels, only the channels $0-3-5$, and only with channel 0 . The retrieved profile for all channels is quite close to the "true" profile (i.e., the temperature used simulating the spectra to be inverted) in between $\sim 50$ and $\sim 80 \mathrm{~km}$, and again close to the "true" temperature at $\sim 30 \mathrm{~km}$. This is the expected behavior based on the sensitivity to the thermal profile displayed by the weighting functions. Also as expected the retrieved profile by inverting only channels $0-3-5$ is nearly identical to the profile from using all channels; the weighting functions for the missing channels only show sensitivity in the troposphere. In that sense, avoiding the use of the MIRO channels with anomalies do not have an impact on the retrieval of the terrestrial atmospheric temperature. For completion the profile retrieved when using only channel 0 is also displayed. In this situation there is no sensitivity to the thermal profile below $\sim 50 \mathrm{~km}$, and the retrieved profile is similar to the a priori profile for the altitudes below.

\subsection{Inversion of ESB2 spectra}

For the inversions of MIRO observed data, we use channels $0-3-5$. The inversion setup is as before. The retrieval characterization is shown in Fig. 10. The averaging kernels $\mathbf{A}$ are displayed for selected altitudes. They peak at the right altitudes between $\sim 50$ and $\sim 85 \mathrm{~km}$, assuring that for that range most of the retrieved information comes from the right altitude. The Full-Width-at-HalfMaximum (FWHM) of the averaging kernels is also plotted. In the atmospheric literature this quantity is used to report the retrieval vertical resolution. For the MIRO observations it is $\sim 10 \mathrm{~km}$. For comparison the EOS-MLS temperature FWHM at $\sim 50 \mathrm{~km}$ is $8 \mathrm{~km}$, although at lower altitudes it can go as low as $\sim 4 \mathrm{~km}$, helped by the limb sounding observation geometry. The retrieval error is plotted in the last panel. The square root of the diagonal of the error matrices $\mathbf{S}_{\epsilon}, \mathbf{S}_{\mathbf{m}}$, and $\mathbf{S}_{\delta}$ described in Section 3.1 are given. The precision (as defined for EOS-MLS) of this MIRO temperature measurements in the mesosphere is $\sim 0.5 \mathrm{~K}$. The total observation error in the mesosphere is $\sim 1 \mathrm{~K}$, but notice that this is likely to be 
an underestimation as other possible instrument related errors (apart from the radiometric noise) have not been characterized. For instance, for limb sounding observations Lossow et al. (2007) showed that instrumental errors (e.g., uncertainty on the radiometric

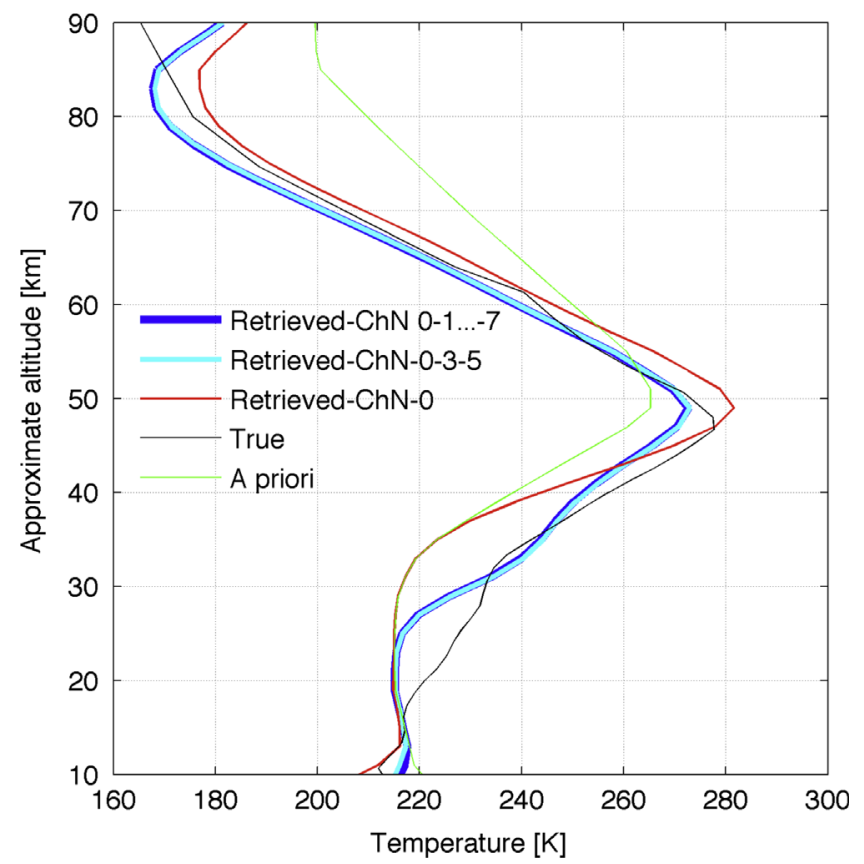

Fig. 9. Temperature retrieval from simulated spectra. Plotted the retrieved temperature when inverting all MIRO channels (blue), channels 0-3-5 (cyan), and channel 0 (red), the temperature profile used to simulate the MIRO spectra (black), and the a priori temperature profile used in the inversions (green). (For interpretation of the references to color in this figure caption, the reader is referred to the web version of this article.) calibration or side band filtering) can also contribute to the temperature retrieval error from the $557 \mathrm{GHz}$ line.

The temperature retrieval is shown in Fig. 11. The MIRO retrieved profile clearly moves away form the a priori temperature and gets very close to the EOS-MLS temperature between $\sim 50$ and $\sim 85 \mathrm{~km}$. As we saw with the inversions of simulated spectra, at lower altitudes the MIRO retrieval mimic the a priori (no sensitivity to the thermal profiles), to get close again to the EOS-MLS profile at $\sim 30 \mathrm{~km}$ (channel 5 adds sensitivity through the image band). To illustrate the variability of the atmospheric temperature, a profile from similarly averaging EOS-MLS observations but from half a year before is also displayed. The spectral residuals (difference between observed spectra and the spectra corresponding to the retrieved atmosphere) are given in Fig. 12. The fitting for channel 0 is reasonable, although some asymmetry around the $557 \mathrm{GHz}$ line (compared with the spectra simulated by ARTS) is visible. For channels 3 and 5 slopes in the observed spectra are also present and cannot be replicated by the radiative transfer simulation. We could speculate that the origin of those differences is instrumental artifacts, but no further conclusions can be derived from just inverting one set of spectra.

Fig. 4 shows that atmospheric emission/absorption from $\mathrm{H}_{2}^{17} \mathrm{O}$, $\mathrm{H}_{2}^{17} \mathrm{O}$, and $\mathrm{CO}$ is expected in channels 1,3 , and 4 , respectively. Different Earth observing instruments also target these species. For instance, at microwave wavelengths EOS-MLS measures CO at $230.53 \mathrm{GHz}$ (Pumphrey et al., 2007), and Odin-SMR measures $\mathrm{H}_{2}^{18} \mathrm{O}$ at $547.67 \mathrm{GHz}$ (Zelinger et al., 2006). In comparison, the lines observed by MIRO are weaker and, being very close to the strong $557 \mathrm{H}_{2} \mathrm{O}$ transition, their expected contribution to the total spectral radiances is very small, as shown in Fig. 4. A possible detection is further complicated by the suspected instrumental artifacts. This can be noticed in the spectral fit of the $\mathrm{H}_{2}^{18} \mathrm{O}$ line in Fig. 12, where the tilt of the spectrum results in brightness temperature variations from one side of the band to the other
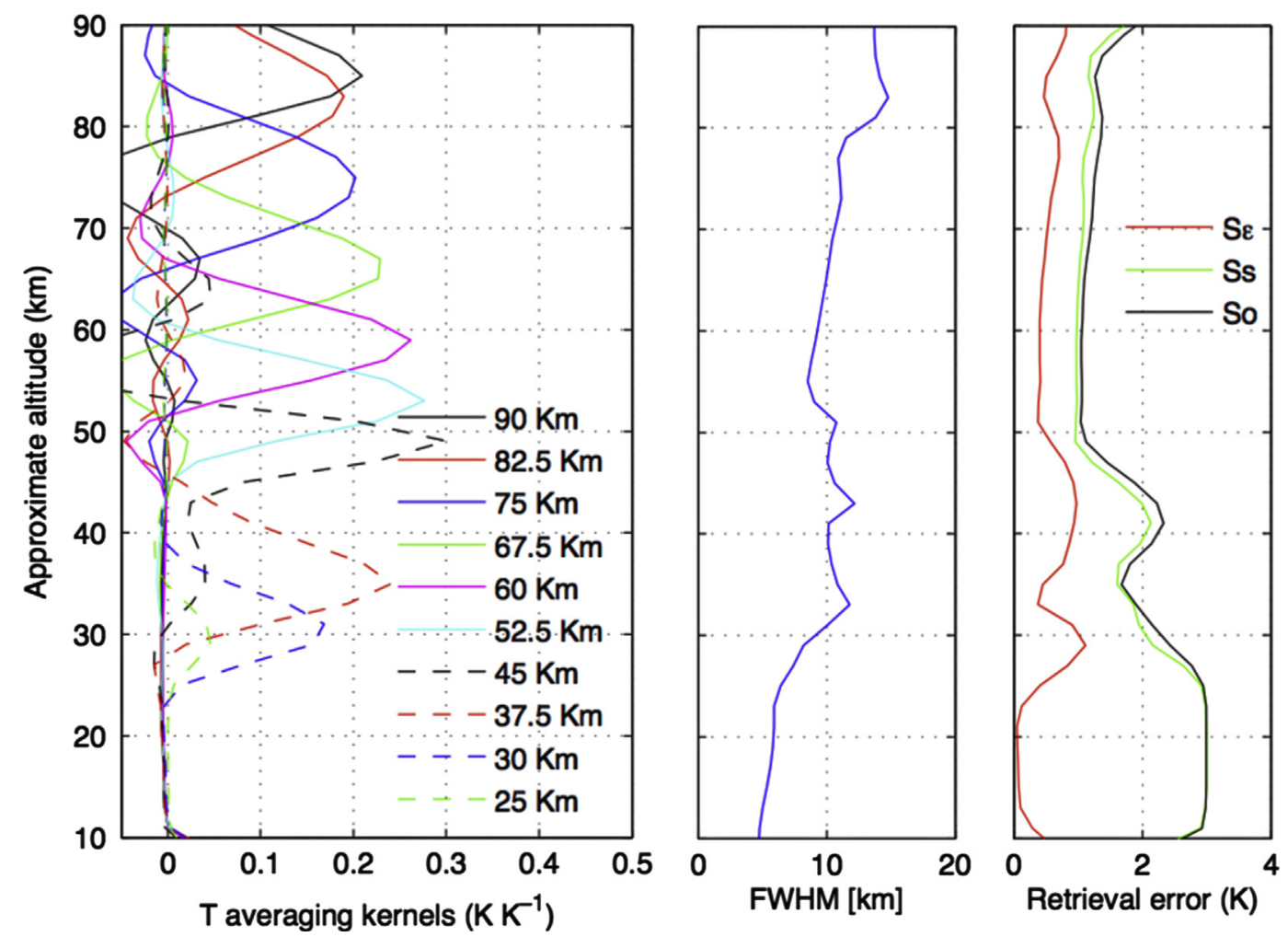

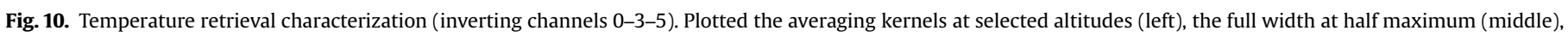

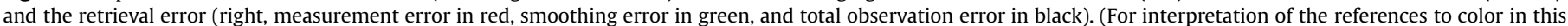
figure caption, the reader is referred to the web version of this article.) 
side as large as the variations simulated by ARTS for this line. Nevertheless, a detection has been attempted by a dedicated onechannel inversion for each of these 3 channels. The EOS-MLS averaged atmosphere for the MIRO flyby is used as a priori

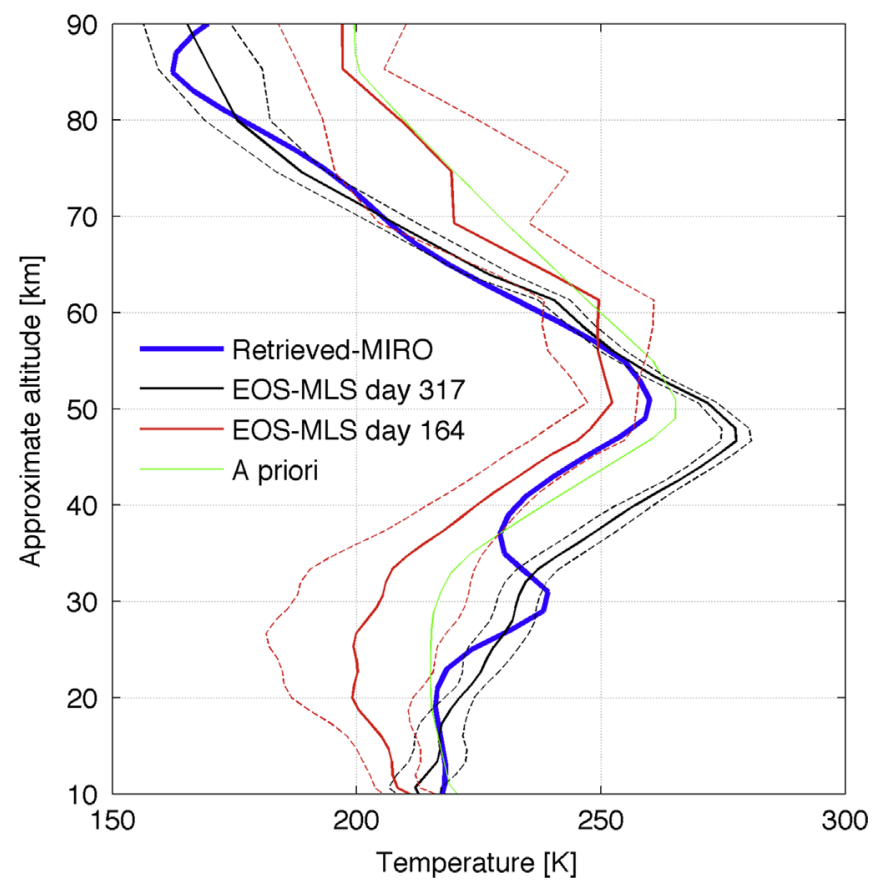

Fig. 11. Temperature retrieval from MIRO channels 0-3-5 observed spectra. Plotted the retrieved temperature (blue), the temperature retrieved from coincident EOSMLS observations during the same day (black), and half a year before (red), and the a priori temperature profile used in the retrieval (green). For the EOS-MLS temperatures the solid line gives the mean temperature profile, and the dashed line \pm one standard deviation around the mean (over the retrievals close to the MIRO flyby ground track). (For interpretation of the references to color in this figure caption, the reader is referred to the web version of this article.) atmosphere for the inversions, and only the relevant species profile and a first-order polynomial fit are retrieved during the inversions. The temperature and/or water vapor are not retrieved as before as these channels considered in isolation have very limited sensitivity. This means that the polynomial fit will try to capture the instrumental artifacts of the spectrum, but also any other deviations between observed and simulated spectrum arising from differences in the "true" and the EOS-MLS atmosphere. The spectral fits are shown in Fig. 13. Once the slope of the spectrum is removed by the polynomial fit, the observed shape of the $\mathrm{H}_{2}^{18} \mathrm{O}$ agrees well with the simulated line. The agreement is not so good for the $\mathrm{H}_{2}^{17} \mathrm{O}$ and $\mathrm{CO}$ lines, although there is some consistency in the shapes of observed and simulated spectra. It should be remembered that these last two channels are affected by the suspected gain instabilities of amplifier A3, making the detection more difficult than for $\mathrm{H}_{2}^{18} \mathrm{O}$. Higher order polynomial fits have been tried for $\mathrm{CO}$ and $\mathrm{H}_{2}^{17} \mathrm{O}$ (results not shown) to see if the could better capture the instrumental artifacts, but no conclusive results have been obtained.

\section{Conclusions}

Sub-millimeter spectra recorded by the MIRO sounder aboard the Rosetta spacecraft have been used at the time of an Earth flyby (November 2007) to check the consistency and validity of the instrumental data. High-resolution spectroscopic data were recorded in 8 channels in the vicinity of the strong water line at $557 \mathrm{GHz}$, and in a broad band continuum channel at $570 \mathrm{GHz}$.

An atmospheric radiative transfer code (ARTS) and standard terrestrial atmospheres have been used to simulate the expected observational results. Comparison of these simulated spectra with the MIRO observations showed that the receiver channels dedicated to observe the $\mathrm{H}_{2}^{17} \mathrm{O}, \mathrm{CO}, \mathrm{NH}_{3}$, and $\mathrm{CH}_{3} \mathrm{OH}(\mathrm{M} 1)$ do not behave as expected. This prompted a review of the inflight technical data for the different flybys, which has confirmed technical anomalies in those four channels. A technical investigation identified the A3 amplifier of
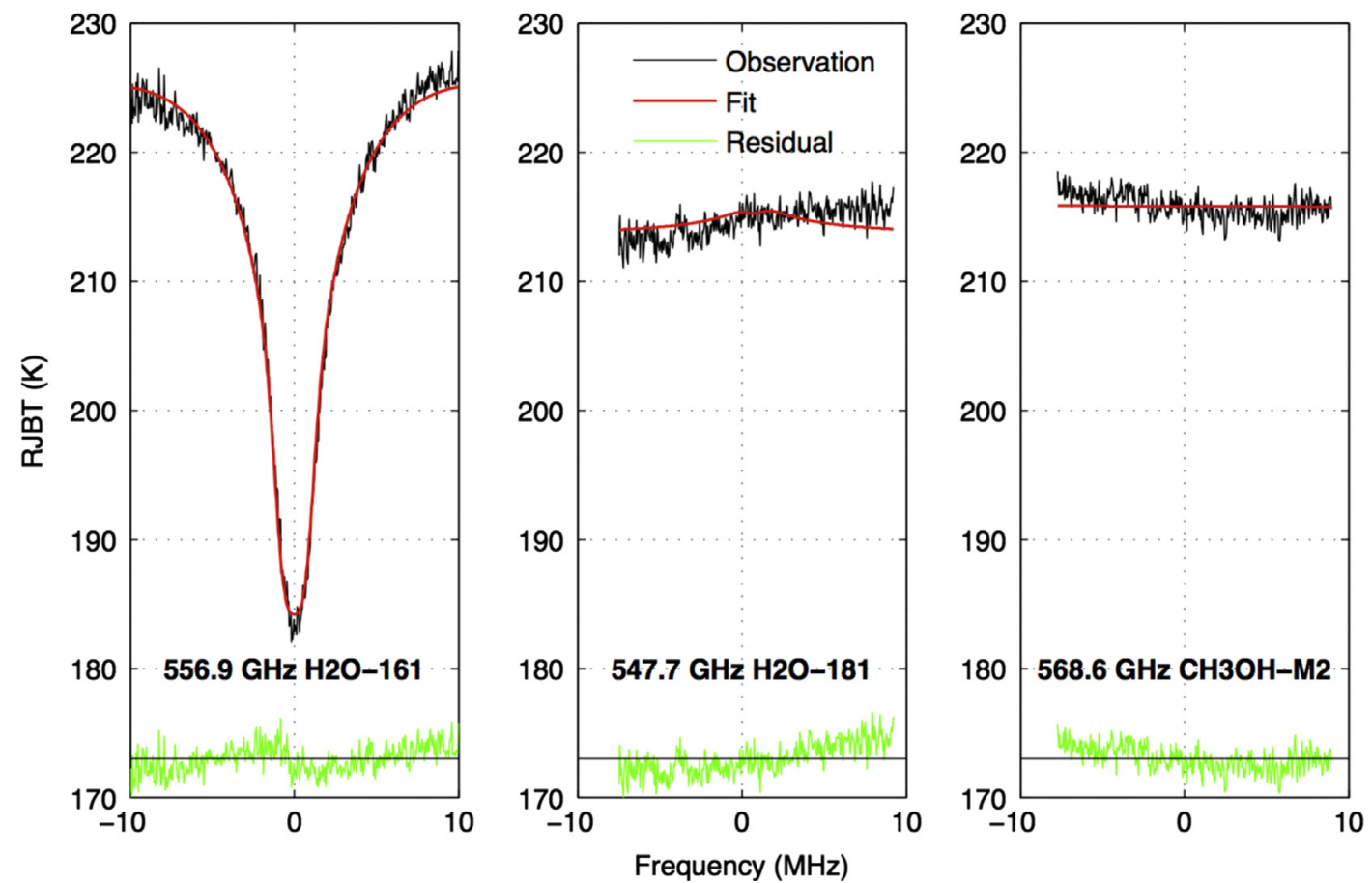

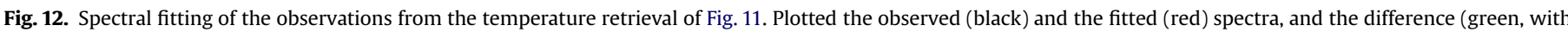

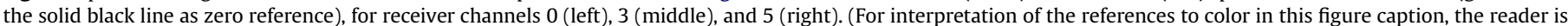
referred to the web version of this article.) 

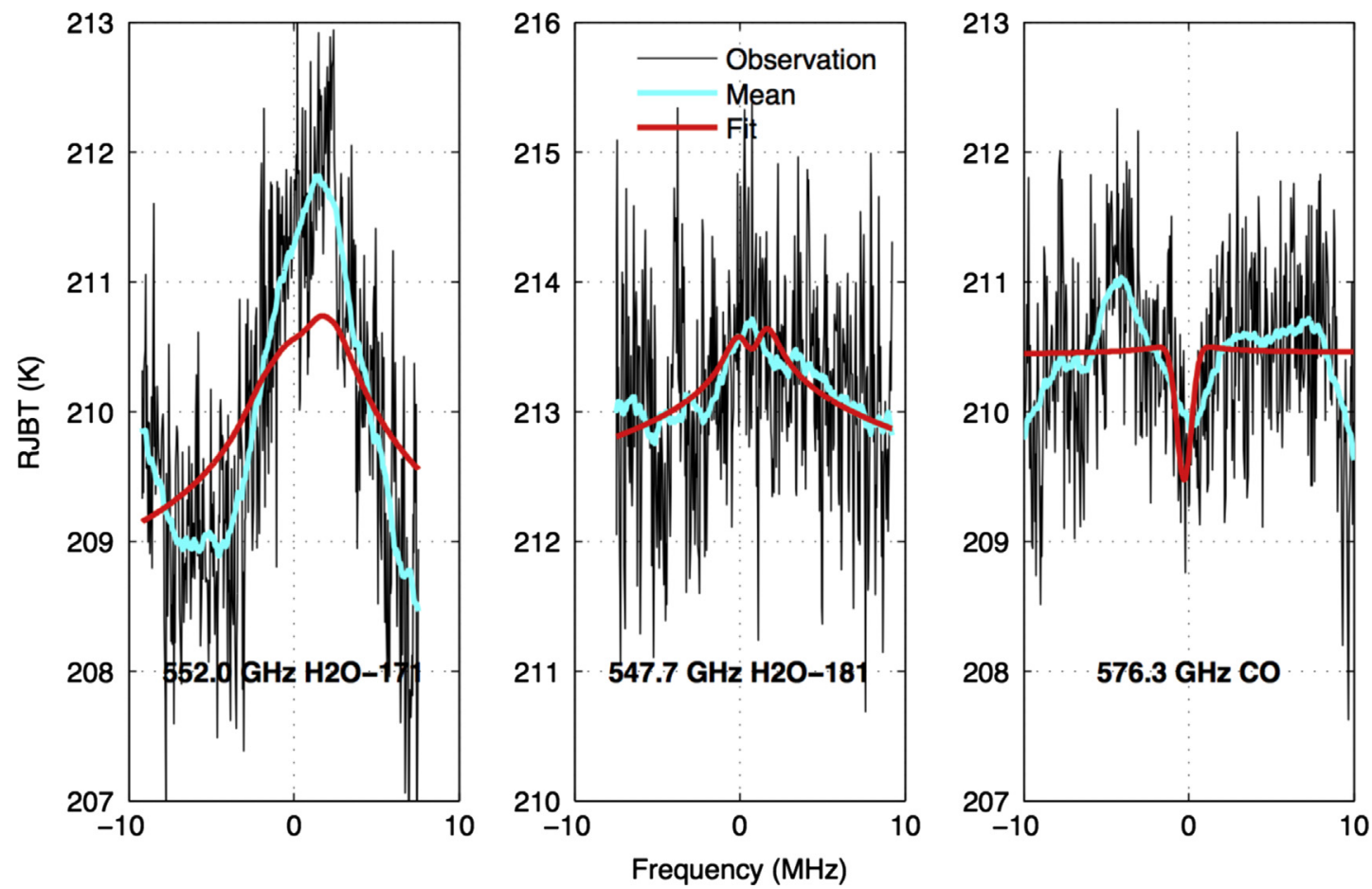

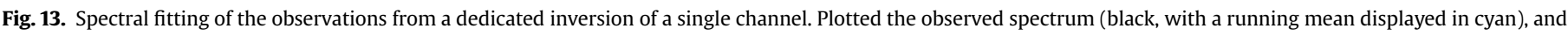

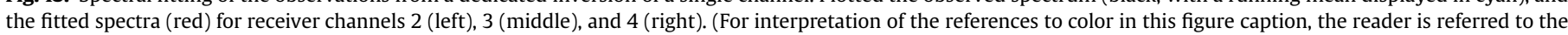
web version of this article.)

the Intermediate Frequency Processor as probably responsible for a large part of the unexpected behavior. Issues with the noise figures of the amplifier have been ruled out; the noise observed in the observations seems nominal and not different from the noise in the channels behaving as expected. On the contrary, relatively large gain variations have been observed for the anomalous channels; this points out to gain instabilities of the $\mathrm{A} 3$ amplifier.

The spectrum from the remaining channels has been further analysed for a retrieval of the atmospheric temperature profile. An optimal estimation inverse model has been implemented by using the atmospheric retrieval tool Qpack. Simulated spectrum has been inverted to illustrate the capability of the MIRO observations to probe the atmospheric thermal profile. Limitations in term of sounding altitude due to the frequency selection of the spectroscopic channels were identified by an analysis of the observations weighting functions, and were also apparent in the retrieved profiles. This was to be expected as MIRO is not optimized for Earth atmospheric sounding, but for cometary studies. The MIRO observations were inverted with the same retrieval setup and compared with the retrieved temperature from the Earth observing instrument EOS-MLS. The MIRO retrieved profile compared well with the retrieved temperature from the $118 \mathrm{GHz}$ observations from EOS-MLS, suggesting a reasonable behavior for the channels not showing clear anomalies. This is further supported by the detection of the much weaker $\mathrm{H}_{2}^{18} \mathrm{O}$ line (compared with the $\mathrm{H}_{2} \mathrm{O}$ line) in one of these channels from a dedicated inversion that included a polynomial fit to remove suspected spectral artefacts.

Physical signals had already been observed during the Steins encounter in the channels suffering anomalies, so these channels are not ruled out for the main mission objective of observing comet 64/P Churyumov Gerasimenko in 2014. Technical solutions to compensate for the anomalous behavior in the affected channels are currently under investigation, and include techniques such as more frequent calibration sequences to assure that the affected channels will still be useful for cometary observations. Once the observations proceed, the quality of these channels can be re-evaluated by checking the consistency in the data gathered from the three $\mathrm{CH}_{3} \mathrm{OH}$ channels, and in the three $\mathrm{H}_{2} \mathrm{O}$ channels. In the case of $\mathrm{CH}_{3} \mathrm{OH}$, the $\mathrm{M} 2$ and $\mathrm{M} 3$ transitions correspond to channels not associated with the A3 amplifier, contrary to the M1 transition. The intensity of the M1 line is intermediate between the ones of the M2 and M3 transitions. Unfortunately, all transitions are weak, and a comparison of the three signals may not be possible at the time of the lander delivery (at an heliocentric distance of $3 \mathrm{AU}$ ), and may be used only in the second part of the mission, when the comet approaches perihelion. A comparison of the signal in the three water channels is expected to be more sensitive. Both $\mathrm{H}_{2}^{16} \mathrm{O}$ and $\mathrm{H}_{2}^{18} \mathrm{O}$ correspond to channels not associated with the $\mathrm{A} 3$ amplifier, contrary to the $\mathrm{H}_{2}^{17} \mathrm{O}$ channel. Numerical simulations (N. Biver, personal communication) show that the weakest water transition $\mathrm{H}_{2}^{17} \mathrm{O}$ is expected to be stronger than the weakest $\mathrm{CH}_{3} \mathrm{OH}$ line (M3), and the comparison of the three water lines could be possible before the time of lander delivery.

\section{Acknowledgments}

We are thankful to Catherine Prigent from the Observatoire de Paris, for constructive discussions concerning this work. We are grateful to the EOS-MLS for making their data accessible. The ARTS community is acknowledged for their efforts toward developing the model. The MIRO Team thanks the ESA Rosetta Project Team, the Rosetta Science Operation Center and the Rosetta Mission Operations Center for their support.

\section{References}

Buehler, S.A., Courcoux, N., John, V.O., 2006. Radiative transfer calculations for a passive microwave satellite sensor: comparing a fast model and a line-by-line model. Journal of Geophysical Research 111. 
Buehler, S.A., Eriksson, P., Kuhn, T., von Engeln, A., Verdes, C., 2004. ARTS, the atmospheric radiative transfer simulator. Journal of Quantitative Spectroscopy and Radiative Transfer 91, 65-93, http://dx.doi.org/10.1016/j.jqsrt.2004.05.051.

Buehler, S.A., Eriksson, P., Lemke, O., 2011. Absorption lookup tables in the radiative transfer model ARTS. Journal of Quantitative Spectroscopy and Radiative Transfer 112 (10), 1559-1567.

Buehler, S.A., John, V.O., Kottayil, A., Milz, M., Eriksson, P., 2010. Efficient radiative transfer simulations for a broadband infrared radiometer - combining a weighted mean of representative frequencies approach with frequency selection by simulated annealing. Journal of Quantitative Spectroscopy and Radiative Transfer 111 (March (4)), 602-615.

Davis, C.P., Evans, K.F., Buehler, S.A., Wu, D.L., Pumphrey, H.C., 2007. 3-D polarised simulations of space-borne passive $\mathrm{mm} / \mathrm{sub}-\mathrm{mm}$ midlatitude cirrus observations: a case study. Atomic and Chemical Physics 7, 4149-4158.

Eriksson, P., Buehler, S.A., Davis, C.P., Emde, C., Lemke, O., 2011. ARTS, the atmospheric radiative transfer simulator, Version 2. Journal of Quantitative Spectroscopy and Radiative Transfer.

Eriksson, P., Chen, D., 2002. Statistical parameters derived from ozonesonde data of importance for passive remote sensing observations of ozone. International Journal of Remote Sensing 23 (22), 4954-4963.

Eriksson, P., Ekström, M., Buehler, S.A., Melsheimer, C., 2006. Efficient forward modelling by matrix representation of sensor responses. International Journal of Remote Sensing 27 (9-10), 1793-1808.

Eriksson, P., Jiménez, C., Buehler, S.A., 2004. Qpack, a tool for instrument simulation and retrieval work. Journal of Quantitative Spectroscopy and Radiative Transfer 91 (1), 47-64.

Gulkis, S., Frerking, M., Crovisier, J., et al., 2007. MIRO: Microwave instrument for Rosetta orbiter. Space Science Reviews 128, 561-597.

Gulkis, S., Keihm, S., Kamp, L., et al., 2010. Millimeter and submillimeter measurements of asteroid (2867) Steins during the Rosetta fly-by. Planetary and Space Science 58, 1077-1087.

Gulkis, S., Keihm, S., Kamp, L., et al., 2012. Continuum and spectroscopic observations of asteroid (21) Lutetia at millimeter and submillimeter wavelengths with the MIRO instrument on the Rosetta spacecraft. Planetary and Space Science 66, 31-42.

Hartogh, P., Hartmann, G., 1990. A high-resolution chirp transform spectrometer for microwave measurements. Measurement Science and Technology 1, 592-595.

Jiménez, C., Buehler, S.A., Rydberg, B., Eriksson, P., Evans, K.F., 2007. Performance simulations for a submillimetre wave cloud ice satellite instrument. Quarterly Journal of the Royal Meteorological Society 133 (S2), 129-149.

Kamp, L., 2012. Report on anomalous behaviour of some CTS bands. Technical Report RO-MIR-TN-2012-001-LWK, Jet Propulsion Laboratory, November.

Lambert, A., et al., 2007. Validation of the Aura Microwave Limb Sounder middle atmosphere water vapor and nitrous oxide measurements. Journal of Geophysical Research 112, D24S36, http://dx.doi.org/10.1029/2007JD008724.

Lossow, S., Urban, J., Eriksson, P., Murtagh, D., Gumbel, J., 2007. Critical parameters for the retrieval of mesospheric water vapour and temperature from Odin/SMR limb measurements at $557 \mathrm{GHz}$. Advances in Space Research 40, 835-845.

Marks, C., Rodgers, C.D., 1993. A retrieval method for atmospheric composition from limb emission measurements. Journal of Geophysical Research 98, 14939-14953.
Melsheimer, C., Verdes, C., Buehler, S.A., Emde, C., Eriksson, P., Feist, D.G., Ichizawa, S., John, V.O., Kasai, Y., Kopp, G., Koulev, N., Kuhn, T., Lemke, O., Ochiai, S., Schreier, F., Sreerekha, T.R., Suzuki, M., Takahashi, C., Tsujimaru, S., Urban, J., 2005. Intercomparison of general purpose clear sky atmospheric radiative transfer models for the millimeter/submillimeter spectral range. Radio Science.

Perrin, A., Puzzarini, C., Colmont, J.-M., Verdes, C., Wlodarczak, G., Cazzoli, G., Buehler, S., Flaud, J.-M., Demaison, J., 2005. Molecular line parameters for the MASTER (millimeter wave acquisitions for stratosphere/troposphere exchange research) database. Journal of Atmospheric Chemistry 50 (June (2)), 161-205.

Pumphrey, H.C., Filipiak, M.J., Livesey, N.J., Schwartz, M.J., Boone, C., Walker, K.A. Bernath, P., Ricaud, P., Barret, B., Clerbaux, C., Jarnot, R.F., Manney, G.L., Waters, J.W. 2007. Validation of middle-atmosphere carbon monoxide retrievals from the Microwave Limb Sounder on Aura. Journal of Geophysical Research 112, D24S38, http://dx.doi.org/10.1029/2007JD008723.

Rodgers, C., 1976. Retrieval of atmospheric temperature and composition from remote measurements of thermal radiation. Reviews of Geophysics and Space Physics 14, 609-624.

Rodgers, C.D., 1990. Characterization and error analysis of profiles retrieved from remote sensing measurements. Journal of Geophysical Research 95, 5587-5595.

Rosenkranz, P., 1993. In: Janssen, M.A. (Ed.), Atmospheric Remote Sensing by Microwave Radiometry. John Wiley and Sons, Inc. (Chapter 2).

Rothman, L., Gordon, I., Barbe, A., Benner, D., Bernath, P., Birk, M., Boudon, V. Brown, L., Campargue, A., Champion, J.-P., Chance, K., Coudert, L., Dana, V., Devi, V., Fally, S., Flaud, J.-M., Gamache, R., Goldman, A., Jacquemart, D., Kleiner, I., Lacome, N., Lafferty, W., Mandin, J.-Y., Massie, S., Mikhailenko, S., Miller, C., Moazzen-Ahmadi, N., Naumenko, O., Nikitin, A., Orphal, J., Perevalov, V., Perrin, A., Predoi-Cross, A., Rinsland, C., Rotger, M., Åimekov, M., Smith, M., Sung, K., Tashkun, S., Tennyson, J., Toth, R., Vandaele, A., Auwera, J.V., 2009. The HITRAN 2008 molecular spectroscopic database. Journal of Quantitative Spectroscopy and Radiative Transfer 110, 533-572.

Schwartz, M.J., et al., 2008. Validation of the Aura Microwave Limb Sounder temperature and geopotential height measurements. Journal of Geophysical Research 113, D15S11, http://dx.doi.org/10.1029/2007JD008783.

Urban, J., Lautier, N., Murtagh, D., et al., 2007. Global observations of middle atmospheric water vapour by the Odin satellite: an overview. Planetary and Space Science 55, 1102-1903.

Verdes, C.L., Buehler, S.A., Perrin, A., Flaud, J.-M., Demaison, J., Wlodarczak, G. Colmont, J.-M., Cazzoli, G., Puzzarini, C., 2005. A sensitivity study on spectroscopic parameter accuracies for a $\mathrm{mm} / \mathrm{sub}-\mathrm{mm}$ limb sounder instrument. Journal of Molecular Spectroscopy 229 (February (2)), 266-275.

von Engeln, A., Buehler, S.A., Langen, J., Wehr, T., Kuenzi, K., 1998. Retrieval of upper stratospheric and mesospheric temperature profiles from Millimeter-Wave Atmospheric Sounder data. Journal of Geophysical Research 103 (D24) 31735-31748.

Waters, J., et al., 2006. The Earth Observing System Microwave Limb Sounder (EOS MLS) on the AURA satellite. IEEE Transactions on Geoscience and Remote Sensing 44, 1075-1092.

Zelinger, Z., Barret, B., Kubát, P., Ricaud, P., Attie, J.-L., Flochmoën, E.L., Urban, J. Murtagh, D., Strizik, M., 2006. Observation of HD18O, CH3OH and vibrationally excited $\mathrm{N}_{2} \mathrm{O}$ from Odin/SMR measurements. Molecular Physics 104 (16-17), 2815-2820. 